\title{
Tight-binding model and direct-gap/indirect-gap transition in single-layer and multilayer $\mathbf{M o S}_{2}$
}

\author{
E. Cappelluti, ${ }^{1,2}$ R. Roldán, ${ }^{1}$ J. A. Silva-Guillén, ${ }^{3}$ P. Ordejón, ${ }^{3}$ and F. Guinea ${ }^{1}$ \\ ${ }^{1}$ Instituto de Ciencia de Materiales de Madrid, CSIC, c/ Sor Juana Ines de la Cruz 3, 28049 Cantoblanco, Madrid, Spain \\ ${ }^{2}$ Istituto dei Sistemi Complessi, U.O.S. Sapienza, CNR, v. dei Taurini 19, 00185 Roma, Italy \\ ${ }^{3}$ Centre d'Investigació en Nanociència i Nanotecnologia-CIN2 (CSIC-ICN), Campus UAB, Bellaterra, Spain
}

(Received 24 April 2013; revised manuscript received 14 July 2013; published 8 August 2013)

\begin{abstract}
In this paper we present a paradigmatic tight-binding model for single-layer as well as multilayered semiconducting $\mathrm{MoS}_{2}$ and similar transition metal dichalcogenides. We show that the electronic properties of multilayer systems can be reproduced in terms of a tight-binding modeling of the single-layer hopping terms by simply adding the proper interlayer hoppings ruled by the chalcogenide atoms. We show that such a tight-binding model makes it possible to understand and control in a natural way the transition between a direct-gap band structure, in single-layer systems, and an indirect gap in multilayer compounds in terms of a momentum/orbital selective interlayer splitting of the relevant valence and conduction bands. The model represents also a suitable playground to investigate in an analytical way strain and finite-size effects.
\end{abstract}

DOI: 10.1103/PhysRevB.88.075409

PACS number(s): 71.15.-m, 73.22.Pr, 63.22.Rc, 68.65.Ac

\section{INTRODUCTION}

The isolation of flakes of single-layer and few-layer graphene $^{1-3}$ has triggered a huge burst of interest in twodimensional layered materials because of their structural and electronic properties. Due to its huge electronic mobility, graphene has been in the last years the main focus of the research in the field. However, a drawback in engineering graphene-based electronic devices is the absence of a gap in the monolayer samples and the difficulty in opening a gap in multilayer systems without affecting the mobility. As an alternative route, recent research is exploring the idea of multilayered heterostructures built up from interfacing different two-dimensional materials. ${ }^{4}$ Along this perspective, semiconducting dichalcogenides such as $\mathrm{MoS}_{2}, \mathrm{MoSe}_{2}, \mathrm{WS}_{2}$, etc., are promising compounds since they can be easily exfoliated and present a suitable small gap in both single-layer and few-layer samples. Quite interestingly, in few-layer $\mathrm{MoS}_{2}$ the size and the nature of the gap depends on the number $N$ of $\mathrm{MoS}_{2}$ layers, with a transition between a direct gap in monolayer $(N=1)$ compounds to a smaller indirect gap for $N \geqslant 2 .^{5-8}$ In addition, the electronic properties appear to be highly sensitive to the external pressure and strain, which affect the insulating gap and, under particular conditions, can also induce an insulator/metal transition. ${ }^{9-20}$ Another intriguing feature of these materials is the strong interplay between the spin and the orbital/valley degrees of freedom, which makes it possible, for instance, to manipulate spins by means of circularly polarized light. ${ }^{21-28}$ Moreover, in $\mathrm{MoSe}_{2}$, a transition between a direct and an indirect gap was observed as a function of temperature. ${ }^{29}$

On the theoretical level, one of the reasons for the strong popularity of graphene is the availability of a paradigmatic Hamiltonian model for the single-layer in terms of few tightbinding (TB) parameters ${ }^{30,31}$ (actually only one, the nearestneighbors carbon-carbon hopping $\gamma_{0}$, in the simplest case). ${ }^{32}$ The well-known Dirac equation can thus be derived from that as a low-energy expansion. Crucial to the development of the theoretical analysis in graphene is also the fact that model Hamiltonians for multilayer graphenes can be built using the single-layer TB description as a fundamental block and just adding additional interlayer hopping terms. ${ }^{33-48}$ Different stacking orders can also be easily investigated. The advantage of such a TB description with respect to first-principles calculations is that it provides a simple starting point for the further inclusion of many-body electron-electron effects by means of quantum field theory (QFT) techniques, as well as of the dynamical effects of the electron-lattice interaction. Tight-binding approaches can be also more convenient than first-principles methods such as density functional theory (DFT) for investigating systems involving a very large number of atoms. Although DFT methods are currently able to handle systems with hundreds or even thousands of atoms, ${ }^{49,50}$ and have been thoroughly applied to large-scale graphene-related problems, ${ }^{51-54}$ they are still computationally challenging and demanding. Therefore, TB has been the method of choice for the study of disordered and inhomogeneous system ${ }^{42,55-67}$ materials nanostructured in large scales (nanoribbons, ripples $)^{68-76}$ or in twisted multilayer materials. ${ }^{77-88}$

While much of the theoretical work of graphenic materials has been based on TB-like approaches, the electronic properties of single-layer and few-layer dichalcogenides have been so far mainly investigated by means of DFT calculations, ${ }^{5,6,8-19,89-95}$ despite early work in nonorthogonal TB models for transition metal dichalcogenides ${ }^{96} \mathrm{Few}$ simplified low-energy Hamiltonian models have been presented for these materials, whose validity is, however, restricted to the specific case of single-layer systems. An effective low-energy model was, for instance, introduced in Refs. 25 and 97 to discuss the spin/orbital/valley coupling at the $\mathrm{K}$ and/or the $\Gamma$ point. Being limited to the vicinity of a specific highsymmetry point, these models cannot be easily generalized to the multilayer case where the gap is indirect with valence and conduction edges located far from the $\mathrm{K}$ point. An effective lattice TB Hamiltonian was, on the other hand, proposed in Ref. 98, valid, in principle, in the whole Brillouin zone. This model considers the spin-orbit coupling, and it includes the effect of a quantizing magnetic field in the spectrum. However, the band structure of the single layer lacks the characteristic second minimum in the conduction band (see later discussion) that will become the effective conduction edge in multilayer systems, so that also in this case the generalization to the 
multilayer compounds is doubtful. In addition, the use of an overlap matrix makes the proposed Hamiltonian unsuitable for a straightforward use as a basis for QFT analyses. This is also the case for a recent model proposed in Ref. 99, where the large number of free fitting parameters and the presence of overlap matrix make such model inappropriate for practical use within the context of QFT.

In this paper we present a suitable TB model for the dichalcogenides valid in both the single-layer case and the multilayer one. Using a Slater-Koster approach, ${ }^{100}$ and focusing on $\mathrm{MoS}_{2}$ as a representative case, we analyze the orbital character of the electronic states at the relevant high-symmetry points. Within this context we show that the transition from a direct gap to an indirect gap in $\mathrm{MoS}_{2}$ as a function of the number of layers can be understood and reproduced in a natural way as a consequence of a momentum/orbital selective interlayer splitting of the main relevant energy levels. In particular, we show that the $p_{z}$ orbital of the $\mathrm{S}$ atoms plays a pivotal role in such transition and it cannot be neglected in reliable TB models aimed to describe single-layer as well as multilayer systems. The TB description here introduced can represent thus the paradigmatic model for the analysis of the electronic properties in multilayer systems in terms of intralayer ligands plus a finite number of interlayer hopping terms. Such a TB model, within the context of the Slater-Koster approach, provides also a suitable tool to include in an analytical and intuitive way effects of pressure/strain by means of the modulation of the interatomic distances. The present analysis defines, in addition, the minimum constraints that the model has to fulfill to guarantee a correct description of the band structure of multilayer compounds.

The paper is structured as follows. In Sec. II we present DFT calculations for single-layer and multilayer (bulk) $\mathrm{MoS}_{2}$, which is here used as a reference for the construction of a TB model. In Sec. III we describe the minimum TB model for the single-layer case needed to reproduce the fundamental electronic properties and the necessary orbital content. The decomposition of the Hamiltonian in blocks and the specific orbital character at the high-symmetry points is discussed. The extension of the TB model to the bulk case, taken as representative of multilayer compounds, is addressed in Sec. IV. We pay special attention to reveal the microscopic origin of the change between a direct-gap to an indirect-gap band structure. In Sec. V we summarize the implications of our analysis in the building of a reliable TB model, and we provide a possible set of TB parameters for the single-layer and multilayer cases.

\section{DFT CALCULATIONS AND ORBITAL CHARACTER}

In the construction of a reliable TB model for semiconducting dichalcogenides we are guided by first-principles DFT calculations that will provide the reference on which to calibrate the TB model. We focus here on $\mathrm{MoS}_{2}$ as a representative case, although we have performed first-principles calculations for comparison also on $\mathrm{WS}_{2}$. The differences in the electronic structure and in the orbital character of these two compounds are, however, minimal and they do not involve any different physics. The structure of single-layer and multilayer $\mathrm{MoS}_{2}$ is depicted in Fig. 1.
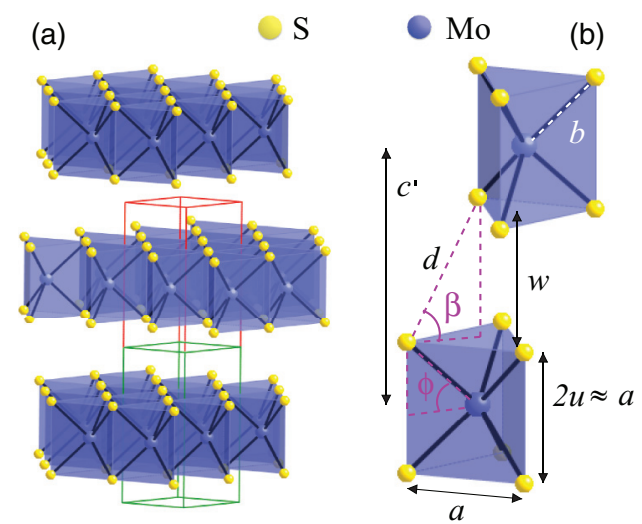

FIG. 1. (Color online) (a) Model of the atomic structure of $\mathrm{MoS}_{2}$. The bulk compound has a $2 \mathrm{H}-\mathrm{MoS}_{2}$ structure with two $\mathrm{MoS}_{2}$ layers per unit cell, each layer being built up from a trigonal prism coordination unit. The small green rectangle represents the unit cell of a monolayer of $\mathrm{MoS}_{2}$, which is doubled (red extension) in the bulk crystal. (b) Detail of the trigonal prisms for the two layers in the bulk compound, showing the lattice constants and the definition of the structural angles used in the text.

The basic unit block is composed of an inner layer of Mo atoms on a triangular lattice sandwiched between two layers of $\mathrm{S}$ atoms lying on the triangular net of alternating hollow sites. Following standard notations, ${ }^{96}$ we denote $a$ as the distance between nearest-neighbor in-plane Mo-Mo and $\mathrm{S}-\mathrm{S}$ distances, $b$ as the nearest-neighbor Mo-S distance, and $u$ as the distance between the Mo and $\mathrm{S}$ planes. The $\mathrm{MoS}_{2}$ crystal forms an almost perfect trigonal prism structure with $b$ and $u$ very close to the their ideal values $b \simeq \sqrt{7 / 12} a$ and $u \simeq a / 2$. In our DFT calculations, we use experimental values for bulk $\mathrm{MoS}_{2},{ }^{96}$ namely $a=3.16 \AA, u=1.586 \AA$, and, in bulk systems, a distance between Mo planes as $c^{\prime}=6.14 \AA$, with a lattice constant in the $2 \mathrm{H}-\mathrm{MoS}_{2}$ structure of $c=2 c^{\prime}$. The in-plane Brillouin zone is thus characterized by the high-symmetry points $\Gamma=(0,0), \mathrm{K}=4 \pi / 3 a(1,0)$, and $\mathrm{M}=4 \pi / 3 a(0, \sqrt{3} / 2)$. DFT calculations are done using the SIESTA code. ${ }^{49,50}$ We use the exchange-correlation potential of Ceperly-Alder ${ }^{101}$ as parametrized by Perdew and Zunger. ${ }^{102}$ We use also a split-valence double- $\zeta$ basis set including polarization functions. ${ }^{103}$ The energy cutoff and the Brillouin zone sampling were chosen to converge the total energy.

The electronic dispersion for the single-layer $\mathrm{MoS}_{2}$ is nowadays well known. We focus only on the block of bands containing the first four conduction bands and by the first seven valence bands, in an energy window of from -7 to $5 \mathrm{eV}$ around the Fermi level. Our DFT calculations are shown in Fig. 2, where we show the orbital character of each band. We use here the shorthand notation $d_{2}$ to denote Mo $4 d_{x^{2}-y^{2}}$, $4 d_{x y}$ orbitals; $d_{1}$ for the Mo $4 d_{x z}, 4 d_{y z}$ orbitals; $d_{0}$ for the Mo $4 d_{3 z^{2}-r^{2}}$ orbital; $p_{x y}$ (or simply $p$ ) to denote the $\mathrm{S} 3 p_{x}, 3 p_{y}$ orbitals; and $p_{z}$ (or simply $z$ ) for the $\mathrm{S} 3 p_{z}$ orbital. The four conduction bands and the seven valence bands are mainly constituted by the five $4 d$ orbitals of Mo and the six (three for each layer) $3 p$ orbitals of $\mathrm{S}$, which sum up to the $93 \%$ of the total orbital weight of these bands.

A special role in the electronic properties of these materials is played by the electronic states labeled as (A)-(D) and 


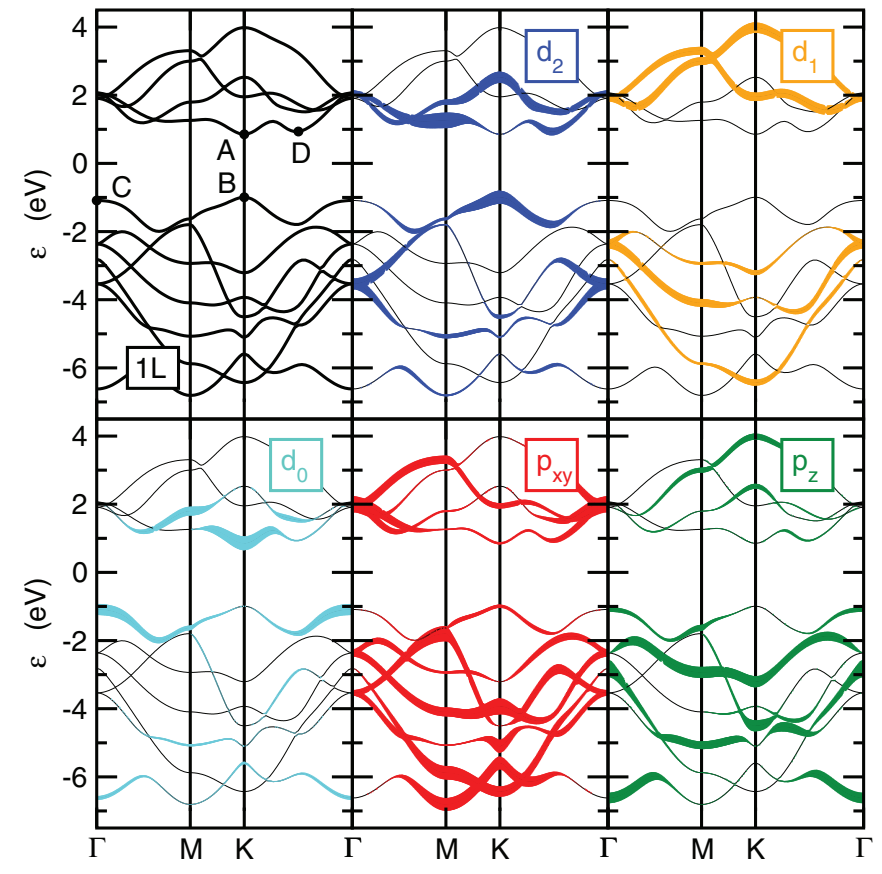

FIG. 2. (Color online) Band structure and orbital character of single-layer $\mathrm{MoS}_{2}$. The top left panel shows the full band structure while, in the other panels, the thickness of the bands represents the orbital weight, where the $d$ character $\left(d_{2}=d_{x^{2}-y^{2}}, d_{x y}, d_{1}=d_{x z}, d_{y z}\right.$, $d_{0}=d_{3 z^{2}-r^{2}}$ ) refers to the Mo atom $4 d$ orbitals, while the $p$ character $\left(p_{x y}=p_{x}, p_{y}\right)$ refers to $2 p$ orbitals of sulfur. marked with black bullets in Fig. 2. A detailed analysis of the orbital character of each energy level at the main high-symmetry points of the Brillouin zone, as calculated by DFT, is provided in Table I. We can notice that an accurate description of the conduction and valence band edges (A)-(B) at the K point involves at least the Mo orbitals $d_{3 z^{2}-r^{2}}, d_{x^{2}-y^{2}}$, $d_{x y}$, and the $\mathrm{S}$ orbitals $p_{x}, p_{y}$. Along this perspective, a five-band TB model, restricted to the subset of these orbitals, was presented in Ref. 98, whereas even the $\mathrm{S} 3 p$ orbitals were furthermore omitted in Ref. 25.

The failure of this latter orbital restriction for a more comprehensive description is, however, pointed out when analyzing other relevant high-symmetry Brillouin points. In particular, concerning the valence band, we can notice a second maximum at the $\Gamma$ point, labeled as (C) in Fig. 2, just $42 \mathrm{meV}$ below the real band edge at the $\mathrm{K}$ point and with main $d_{0}-p_{z}$ orbital character. The relevance of this secondary band extreme is evident in the multilayer compounds $(N \geqslant 2)$, where such maximum at $\Gamma$ increases its energy to become the effective band edge. ${ }^{5,8}$

The band structure with the orbital character for the bulk $(N=\infty)$ case, representative of the multilayer case, is shown in Fig. 3. A similar change of the topology of the band edge occurs in the conduction band. Here a secondary minimum, labeled as D in Fig. 2, at $\mathrm{Q}=4 \pi / 3 a(1 / 2,0)$, midway along the $\Gamma-\mathrm{K}$ cut, is present in the single-layer compounds. Such minimum, however, moves down in energy in multilayer systems to become the effective conduction band edge. ${ }^{5,8}$

TABLE I. Energy levels and orbital content of single-layer $\mathrm{MoS}_{2}$ evaluated by DFT calculations. We report here the first two main orbital characters belonging to the blocks Mo- $4 d$ and S-3p, while the following column shows the remaining character not belonging to these orbital group. Also show is the association of each level with the corresponding eigenvalue of the TB model and the symmetry with respect to the $z \rightarrow-z$ inversion ( $\mathrm{E}=$ even, $\mathrm{O}=$ odd). The label $E_{\alpha \beta, \pm}$ in the last column denotes the orbital character of the TB eigenstate, with $\alpha, \beta=p, z, d_{2}, d_{1}, d_{0}$, where $p=p_{x}, p_{y}, z=p_{z}, d_{2}=d_{x^{2}-y^{2}}, d_{x y}, d_{1}=d_{x z}, d_{y z}, d_{0}=d_{3 z^{2}-r^{2}}$. The index \pm denotes the higher energy $[(+)=$ antibonding] and the lower energy $[(-)=$ bonding].

\begin{tabular}{|c|c|c|c|c|c|}
\hline $\begin{array}{l}\text { Energy } \\
\text { DFT (eV) }\end{array}$ & $\begin{array}{l}\text { Main } \\
\text { orbital }\end{array}$ & $\begin{array}{c}\text { Second } \\
\text { orbital }\end{array}$ & $\begin{array}{c}\text { Other } \\
\text { orbitals }\end{array}$ & Symmetry & $\begin{array}{c}\text { TB } \\
\text { label }\end{array}$ \\
\hline \multicolumn{6}{|c|}{$\Gamma$ point } \\
\hline $2.0860^{\mathrm{a}}$ & $68 \% p_{x / y}$ & $29 \% d_{2}$ & $3 \%$ & $\mathrm{E}$ & $E_{p d_{2},+}(\Gamma)$ \\
\hline $1.9432^{\mathrm{a}}$ & $58 \% p_{x / y}$ & $36 \% d_{1}$ & $6 \%$ & $\mathrm{O}$ & $E_{p d_{1},+}(\Gamma)$ \\
\hline-1.0341 & $66 \% d_{0}$ & $28 \% p_{z}$ & $6 \%$ & $\mathrm{E}$ & $E_{z d_{0},+}(\Gamma)$ \\
\hline-2.3300 & $54 \% d_{1}$ & $42 \% p_{x / y}$ & $4 \%$ & $\mathrm{O}$ & $E_{p d_{1},-}(\Gamma)$ \\
\hline-2.6801 & $100 \% p_{z}$ & & $0 \%$ & $\mathrm{O}$ & $E_{z}(\Gamma)$ \\
\hline$-3.4869^{a}$ & $65 \% d_{2}$ & $32 \% p_{x / y}$ & $3 \%$ & $\mathrm{E}$ & $E_{p d_{2},-}(\Gamma)$ \\
\hline-6.5967 & $57 \% p_{z}$ & $23 \% d_{0}$ & $20 \%$ & $\mathrm{E}$ & $E_{z d_{0},-}(\Gamma)$ \\
\hline \multicolumn{6}{|c|}{$\mathrm{K}$ point } \\
\hline 4.0127 & $60 \% d_{1}$ & $36 \% p_{z}$ & $4 \%$ & $\mathrm{O}$ & $E_{z d_{1},+}(\mathrm{K})$ \\
\hline 2.5269 & $65 \% d_{2}$ & $29 \% p_{z}$ & $6 \%$ & $\mathrm{E}$ & $E_{z d_{2},+}(\mathrm{K})$ \\
\hline 1.9891 & $50 \% d_{1}$ & $31 \% p_{x / y}$ & $19 \%$ & $\mathrm{O}$ & $E_{p d_{1},+}(\mathrm{K})$ \\
\hline 0.8162 & $82 \% d_{0}$ & $12 \% p_{x / y}$ & $6 \%$ & $\mathrm{E}$ & $E_{p d_{0},+}(\mathrm{K})$ \\
\hline-0.9919 & $76 \% d_{2}$ & $20 \% p_{x / y}$ & $4 \%$ & $\mathrm{E}$ & $E_{p d_{2},+}(\mathrm{K})$ \\
\hline-3.1975 & $67 \% p_{z}$ & $27 \% d_{1}$ & $6 \%$ & $\mathrm{O}$ & $E_{z d_{1},-}(\mathrm{K})$ \\
\hline-3.9056 & $85 \% p_{x / y}$ & & $15 \%$ & $\mathrm{O}$ & $E_{p}(\mathrm{~K})$ \\
\hline-4.5021 & $65 \% p_{z}$ & $25 \% d_{2}$ & $10 \%$ & $\mathrm{E}$ & $E_{z d_{2},-}(\mathrm{K})$ \\
\hline-5.0782 & $71 \% p_{x / y}$ & $12 \% d_{2}$ & $17 \%$ & $\mathrm{E}$ & $E_{p d_{2},-}(\mathrm{K})$ \\
\hline-5.5986 & $66 \% p_{x / y}$ & $14 \% d_{0}$ & $20 \%$ & $\mathrm{E}$ & $E_{p d_{0},-}(\mathrm{K})$ \\
\hline-6.4158 & $60 \% p_{x / y}$ & $37 \% d_{1}$ & $3 \%$ & $\mathrm{O}$ & $E_{p d_{1},-}(\mathrm{K})$ \\
\hline
\end{tabular}

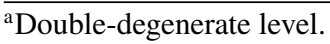




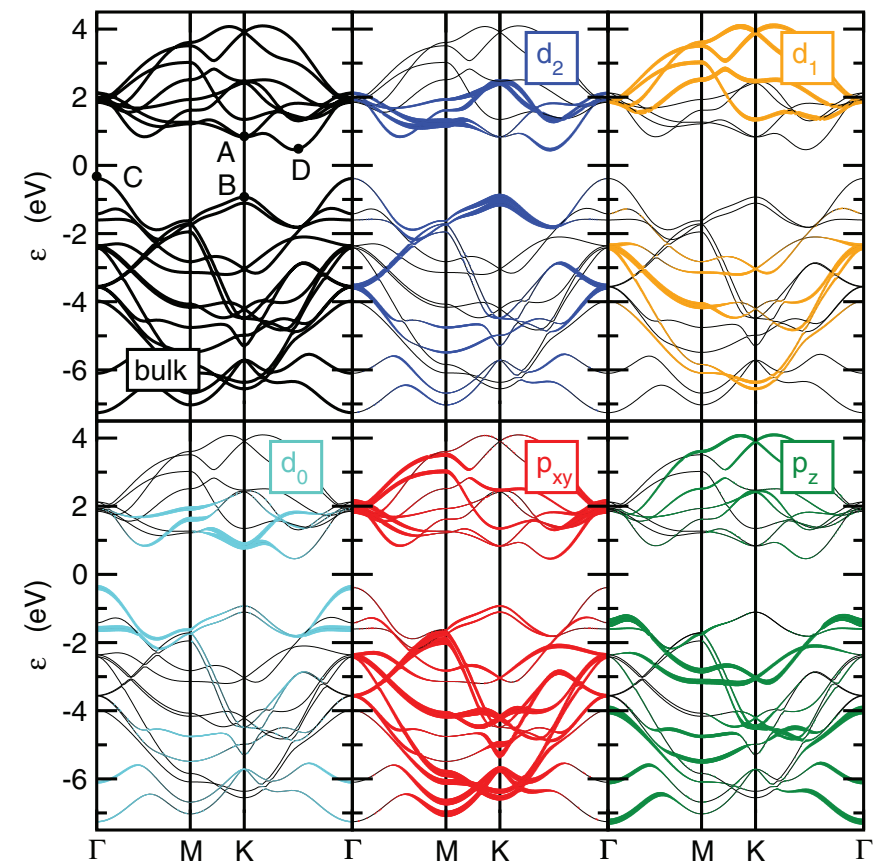

FIG. 3. (Color online) Band structure and orbital character for bulk $2 \mathrm{H}-\mathrm{MoS}_{2}$. Labels similar as in Fig. 2.

Even in this case, a relevant $p_{z}$ component is involved in the orbital character of this electronic state. The topological changes of the location of the band edges in the Brillouin zone are responsible for the observed switch from a direct to an indirect gap in multilayer samples. As we will see, thus, the inclusion of the $p_{z}$ orbitals in the full TB Hamiltonian is not only desirable for a more complete description, but it is also unavoidable to understand the evolution of the band structure as a function of the number of layers.

\section{TIGHT-BINDING DESCRIPTION OF THE SINGLE LAYER}

The aim of this section is to define a TB model for the single layer which will be straightforwardly generalizable to the multilayer case by adding the appropriate interlayer hopping. We show that, to this purpose, all the $4 d$ Mo orbitals and the $3 p \mathrm{~S}$ orbitals are needed to be taken into account. Considering that the unit cell contains two $\mathrm{S}$ atoms, we define the Hilbert space by means of the 11-fold vector,

$$
\begin{array}{r}
\phi_{i}^{\dagger}=\left(p_{i, x, t}^{\dagger}, p_{i, y, t}^{\dagger}, p_{i, z, t}^{\dagger}, d_{i, 3 z^{2}-r^{2}}^{\dagger}, d_{i, x^{2}-y^{2}}^{\dagger},\right. \\
\left.d_{i, x y}^{\dagger}, d_{i, x z}^{\dagger}, d_{i, y z}^{\dagger}, p_{i, x, b}^{\dagger}, p_{i, y, b}^{\dagger}, p_{i, z, b}^{\dagger}\right),
\end{array}
$$

where $d_{i, \alpha}$ creates an electron in the orbital $\alpha$ of the Mo atom in the $i$-unit cell, $p_{i, \alpha, t}$ creates an electron in the orbital $\alpha$ of the top ( $t$ ) layer atom $\mathrm{S}$ in the $i$-unit cell, and $p_{i, \alpha, b}$ creates an electron in the orbital $\alpha$ of the bottom $(b)$ layer atom $S$ in the $i$-unit cell.

Once the Hilbert space has been introduced, the TB model is defined by the hopping integrals between the different orbitals, described, in the framework of a Slater-Koster description, in terms of $\sigma, \pi$, and $\delta$ ligands. ${ }^{100}$ In order to provide a TB model as a suitable basis for the inclusion of many-body effects by means of diagrammatic techniques, we assume that the basis orbitals are orthonormal, so that the overlap matrix is the unit matrix. A preliminary analysis based on the interatomic distance can be useful to identify the most relevant hopping processes. In particular, these are expected to be the ones between nearest-neighbor Mo-S (interatomic distances $b=2.41 \AA$ ), between the nearest-neighbor in-plane Mo-Mo, and between the nearest-neighbor in-plane and out-of-plane S-S atoms (interatomic distance $a=3.16 \AA$ ). Further distant atomic bonds, in single-layer systems, start from hopping between second-nearest-neighbor Mo-S atoms, with interatomic distance $3.98 \AA$, and they will be here discarded.

All the hopping processes of the relevant pair of neighbors are described in terms of the Slater-Koster parameters, respectively, $V_{p d \sigma}, V_{p d \pi}$ (Mo-S bonds), $V_{d d \sigma}, V_{d d \pi}, V_{d d \delta}$ (Mo-Mo bonds), and $V_{p p \sigma}, V_{p p \pi}$ (S-S bonds). Additional relevant parameters are the crystal fields $\Delta_{0}, \Delta_{1}, \Delta_{2}, \Delta_{p}, \Delta_{z}$, describing, respectively, the atomic level $l=0\left(d_{3 z^{2}-r^{2}}\right), l=1$ $\left(d_{x z}, d_{y z}\right)$, and $l=2\left(d_{x^{2}-y^{2}}, d_{x y}\right)$ Mo orbitals, the in-plane $\left(p_{x}\right.$, $\left.p_{y}\right) \mathrm{S}$ orbitals, and of the out-of-plane $p_{z} \mathrm{~S}$ orbitals. We end up with a total of $12 \mathrm{~TB}$ parameters to be determined, namely, $\Delta_{0}, \Delta_{1}, \Delta_{2}, \Delta_{p}, \Delta_{z}, V_{d d \sigma}, V_{d d \pi}, V_{d d \delta}, V_{p p \sigma}, V_{p p \pi}, V_{p d \sigma}$, and $V_{p d \pi}$.

In the orbital basis of Eq. (1), we can write thus the TB Hamiltonian in the form

$$
H=\sum_{\mathbf{k}} \phi_{\mathbf{k}}^{\dagger} \hat{H}_{\mathbf{k}} \phi_{\mathbf{k}},
$$

where $\phi_{\mathbf{k}}$ is the Fourier transform of $\phi_{i}$ in momentum space. The Hamiltonian matrix can be written (we drop for simplicity from now on the index $\mathbf{k}$ ) as

$$
\hat{H}=\left(\begin{array}{ccc}
\hat{H}_{p t, p t} & \hat{H}_{d, p t}^{\dagger} & \hat{H}_{p t, p b} \\
\hat{H}_{d, p t} & \hat{H}_{d, d} & \hat{H}_{d, p b} \\
\hat{H}_{p b, p b}^{*} & \hat{H}_{d, p b}^{\dagger} & \hat{H}_{p b, p b}
\end{array}\right),
$$

where $\hat{H}_{p b, p b}=\hat{H}_{p t, p t}$ describes the in-plane hopping in the top and bottom $\mathrm{S}$ layer, namely,

$$
\hat{H}_{p b, p b}=\hat{H}_{p t, p t}=\left(\begin{array}{ccc}
H_{x / x} & H_{x / y} & 0 \\
H_{x / y}^{*} & H_{y / y} & 0 \\
0 & 0 & H_{z / z}
\end{array}\right),
$$

$\hat{H}_{d, d}$ describes the in-plane hopping in the middle Mo layer, namely,

$$
\hat{H}_{d, d}=\left(\begin{array}{ccccc}
H_{z^{2} / z^{2}} & H_{z^{2} / x^{2}} & H_{z^{2} / x y} & 0 & 0 \\
H_{z^{2} / x^{2}}^{*} & H_{x^{2} / x^{2}} & H_{x^{2} / x y} & 0 & 0 \\
H_{z^{2} / x y}^{*} & H_{x^{2} / x y}^{*} & H_{x y / x y} & 0 & 0 \\
0 & 0 & 0 & H_{x z / x z} & H_{x z / y z} \\
0 & 0 & 0 & H_{x z / y z}^{*} & H_{y z / y z}
\end{array}\right),
$$

$\hat{H}_{p t, p b}$ describes the vertical hopping between S orbitals in the top and bottom layer,

$$
\hat{H}_{p t, p b}=\left(\begin{array}{ccc}
V_{p p \pi} & 0 & 0 \\
0 & V_{p p \pi} & 0 \\
0 & 0 & V_{p p \sigma}
\end{array}\right),
$$


and $\hat{H}_{d, p t}, \hat{H}_{d, p b}$ describes the hopping between Mo and $\mathrm{S}$ atoms in the top and bottom planes, respectively,

$$
\begin{gathered}
\hat{H}_{d, p t}=\left(\begin{array}{ccc}
H_{z^{2} / x} & H_{z^{2} / y} & H_{z^{2} / z} \\
H_{x^{2} / x} & H_{x^{2} / y} & H_{x^{2} / z} \\
H_{x y / x} & H_{x y / y} & H_{x y / z} \\
H_{x z / x} & H_{x z / y} & H_{x z / z} \\
H_{y z / x} & H_{y z / y} & H_{y z / x}
\end{array}\right), \\
\hat{H}_{d, p t}=\left(\begin{array}{ccc}
H_{z^{2} / x} & H_{z^{2} / y} & -H_{z^{2} / z} \\
H_{x^{2} / x} & H_{x^{2} / y} & -H_{x^{2} / z} \\
H_{x y / x} & H_{x y / y} & -H_{x y / z} \\
-H_{x z / x} & -H_{x z / y} & H_{x z / z} \\
-H_{y z / x} & -H_{y z / y} & H_{y z / x}
\end{array}\right) .
\end{gathered}
$$

Here and in the following, for the sake of compactness, we use the shorthand notation $3 z^{2}-r^{2} \Rightarrow z^{2}$ and $x^{2}-y^{2} \Rightarrow x^{2}$. An explicit expression for the different Hamiltonian matrix elements in terms of the Slater-Koster TB parameters can be provided following the seminal work by Doran et al. ${ }^{104}$ and it is reported for completeness in Appendix A.

Equations (2)-(8) define our TB model in terms of an $11 \times 11$ Hamiltonian $\hat{H}$ which can be now explicitly solved to get eigenvalues and eigenvectors in the whole Brillouin zone or along the main axes of high symmetry. It is now an appealing task to associate each DFT energy level with the Hamiltonian eigenvalues, whose eigenvectors will shed light on the properties of the electronic states. Along this line, we are facilitated by symmetry arguments which make it possible, in the monolayer compounds, to decouple the $11 \times 11$ Hamiltonian in Eq. (3) in two main blocks, with different symmetry with respect to the mirror inversion $z \rightarrow$ $-z .{ }^{104}$ This task is accomplished by introducing a symmetric and antisymmetric linear combination of the $p$ orbital of the $\mathrm{S}$ atoms on the top/bottom layers. More explicitly, we use the basis vector

$$
\begin{gathered}
\tilde{\phi}_{k}^{\dagger}=\left(d_{k, 3 z^{2}-r^{2}}^{\dagger}, d_{k, x^{2}-y^{2}}^{\dagger}, d_{k, x y}^{\dagger}, p_{k, x, S}^{\dagger}, p_{k, y, S}^{\dagger}, p_{k, z, A}^{\dagger},\right. \\
\left.d_{k, x z}^{\dagger}, d_{k, y z}^{\dagger}, p_{k, x, A}^{\dagger}, p_{k, y, A}^{\dagger}, p_{k, z, S}^{\dagger}\right),
\end{gathered}
$$

where $\quad p_{k, \alpha, S}^{\dagger}=\left(p_{k, \alpha, t}^{\dagger}+p_{k, \alpha, b}^{\dagger}\right) / \sqrt{2}, \quad p_{k, \alpha, A}^{\dagger}=\left(p_{k, \alpha, t}^{\dagger}-\right.$ $\left.p_{k, \alpha, b}^{\dagger}\right) / \sqrt{2}$. Note that our basis differs slightly with respect to the one employed in Ref. 104 because we have introduced explicitly the proper normalization factors to make it unitary. In this basis we can write thus

$$
\hat{H}=\left(\begin{array}{cc}
\hat{H}_{\mathrm{E}} & 0 \\
0 & \hat{H}_{\mathrm{O}}
\end{array}\right),
$$

where $\hat{H}_{\mathrm{E}}$ is a $6 \times 6$ block with even $(\mathrm{E})$ symmetry with respect to the mirror inversion $z \rightarrow-z$, and $\hat{H}_{\mathrm{O}}$ is a $5 \times 5$ block with odd (O) symmetry. We should remark, however, that such decoupling holds true only in the single-layer case and only in the absence of a $z$-axis electric field, as can be induced by substrates or under gating conditions. In the construction of a TB model that could permit a direct generalization to the multilayer case, the interaction between the band blocks with even and odd symmetry should be thus explicitly retained.

The association between DFT energy levels and TB eigenstates is now further simplified on specific high-symmetry points of the Brillouin zone. Most important are the $\mathrm{K}$ and the $\Gamma$ points, which are strictly associated with the direct and indirect gap in monolayer and multilayered compounds.

\section{A. $\Gamma$ point}

We present here a detailed analysis of the eigenstates and their orbital character at the $\Gamma$ point. For the sake of simplicity, we discuss separately the blocks with even and odd symmetry with respect to the inversion $z \rightarrow-z$. The identification of the DFT levels with the TB eigenstates is facilitated by the possibility of decomposing the full Hamiltonian in smaller blocks, with typical size $2 \times 2$ (dimers) or $1 \times 1$ (monomers). In particular, the $6 \times 6$ block with even symmetry can be decomposed (see Appendix B for details) as

$$
\hat{H}_{\mathrm{E}}(\Gamma)=\left(\begin{array}{ccc}
\hat{H}_{z d_{0}}(\Gamma) & 0 & 0 \\
0 & \hat{H}_{p d_{2}}(\Gamma) & 0 \\
0 & 0 & \hat{H}_{p d_{2}}(\Gamma)
\end{array}\right) .
$$

Here each matrix $\hat{H}_{p d_{2}}, \hat{H}_{z d_{0}}$ represents a $2 \times 2$ block where the indices describe the orbital character of the dimer. In particular, $\hat{H}_{p d_{2}}$ involves only $d_{2}=d_{x^{2}-y^{2}}, d_{x y}$ Mo orbitals and $p_{x}, p_{y}$ $\mathrm{S}$ orbitals, whereas $\hat{H}_{z d_{0}}$ involves only the $d_{0}=d_{3 z^{2}-r^{2}}$ Mo orbital and the $p_{z} \mathrm{~S}$ orbital. As it is evident in (11), the block $\hat{H}_{p d_{2}}$ appears twice and it is thus doubly degenerate. Similarly, we have

$$
\hat{H}_{\mathrm{O}}(\Gamma)=\left(\begin{array}{ccc}
\hat{H}_{p d_{1}}(\Gamma) & 0 & 0 \\
0 & \hat{H}_{p d_{1}}(\Gamma) & 0 \\
0 & 0 & \Gamma_{z}^{\mathrm{O}}
\end{array}\right),
$$

where the doubly degenerate block $\hat{H}_{p d_{1}}$ involves only $d_{1}=$ $d_{x z}, d_{y z}$ Mo orbitals and $p_{x}, p_{y} \mathrm{~S}$ orbitals, while $\Gamma_{z}^{\mathrm{O}}$ is a $1 \times 1$ block (monomer) with pure character $p_{z}$.

It is also interesting to give a closer look at the inner structure of a generic Hamiltonian sub-block. Considering, for instance, $\hat{H}_{z d_{0}}$ as an example, we can write

$$
\hat{H}_{z d_{0}}(\Gamma)=\left(\begin{array}{cc}
\Gamma_{0} & \sqrt{2} \Gamma_{z d_{0}} \\
\sqrt{2} \Gamma_{z d_{0}} & \Gamma_{z}^{\mathrm{E}}
\end{array}\right),
$$

where $\Gamma_{0}$ is an energy level with pure Mo $d_{0}$ orbital character and $\Gamma_{z}^{\mathrm{E}}$ is an energy level with pure $\mathrm{S} p_{z}$ orbital character. Thus, the off-diagonal term $\sqrt{2} \Gamma_{z d_{0}}$ acts here as a "hybridization," mixing the pure orbital characters of $\Gamma_{0}$ and $\Gamma_{z}^{\mathrm{E}}$. The suffix "E" here reminds that the level $\Gamma_{z}^{\mathrm{E}}$ belongs to the even symmetry block, and it is useful to distinguish this state from a similar one with odd symmetry (and different energy). Keeping $\hat{H}_{z d_{0}}$ as an example, the eigenvalues of a generic $2 \times 2$ block can be obtained analytically:

$$
E_{z d_{0}, \pm}(\Gamma)=\frac{\Gamma_{0}+\Gamma_{z}^{\mathrm{E}}}{2} \pm \sqrt{\left(\frac{\Gamma_{0}-\Gamma_{z}^{\mathrm{E}}}{2}\right)^{2}+2 \Gamma_{z d_{0}}^{2}} .
$$

The explicit expressions of $\Gamma_{\alpha}$ and $\Gamma_{\alpha \beta}$ in terms of the Slater-Koster TB parameters are reported in Appendix A.

It is interesting to note that the diagonal terms $\Gamma_{\alpha}(\alpha=$ $\left.d_{0}, d_{1}, d_{2}, p, z\right)$ are purely determined by the crystal fields $\Delta_{\alpha}$ and by the TB parameters $V_{d d \sigma}, V_{d d \pi}, V_{d d \delta}, V_{p p \sigma}, V_{p p \pi}$, connecting Mo-Mo and S-S atoms, whereas the hybridization 
off-diagonal terms $\Gamma_{\alpha \beta}$ depend exclusively on the Mo-S nearest-neighbor hopping $V_{p d \sigma}, V_{p d \pi}$.

A careful comparison between the orbital character of each eigenvector with the DFT results makes it possible now to identify in an unambiguous way each DFT energy level with its analytical TB counterpart. Such an association is reported in Table I, where also the even/odd symmetry inversion is considered.

The use of the present analysis to characterize the properties of the multilayer $\mathrm{MoS}_{2}$ is discussed in Sec. IV.

\section{B. K point}

A crucial role in the properties of semiconducting dichalcogenides is played by the $\mathrm{K}$ point in the Brillouin zone, where the direct semiconducting gap occurs in the single-layer systems. The detailed analysis of the electronic spectrum is also favored here by the possibility of reducing the full $11 \times 11$ Hamiltonian in smaller sub-blocks. This feature is, however, less evident than at the $\Gamma$ point. The even and odd components of the Hamiltonian take the form

$$
\begin{gathered}
\hat{H}_{\mathrm{E}}(\mathrm{K})=\left(\begin{array}{cccccc}
K_{0} & 0 & 0 & -i \sqrt{2} K_{p d_{0}} & \sqrt{2} K_{p d_{0}} & 0 \\
0 & K_{2} & 0 & i \sqrt{2} K_{p d_{2}} & \sqrt{2} K_{p d_{2}} & \sqrt{2} K_{z d_{2}} \\
0 & 0 & K_{2} & -\sqrt{2} K_{p d_{2}} & i \sqrt{2} K_{p d_{2}} & -i \sqrt{2} K_{z d_{2}} \\
i \sqrt{2} K_{p d_{0}} & -i \sqrt{2} K_{p d_{2}} & -\sqrt{2} K_{p d_{2}} & K_{p}^{\mathrm{E}} & 0 & 0 \\
\sqrt{2} K_{p d_{0}} & \sqrt{2} K_{p d_{2}} & -i \sqrt{2} K_{p d_{2}} & 0 & K_{p}^{\mathrm{E}} & 0 \\
0 & \sqrt{2} K_{z d_{2}} & i \sqrt{2} K_{z d_{2}} & 0 & 0 & K_{z}^{\mathrm{E}}
\end{array}\right), \\
\hat{H}_{\mathrm{O}}(\mathrm{K})=\left(\begin{array}{ccccc}
K_{1} & 0 & \sqrt{2} K_{p d_{1}} & -i \sqrt{2} K_{p d_{1}} & -i \sqrt{2} K_{z d_{1}} \\
0 & K_{1} & -i \sqrt{2} K_{p d_{1}} & -\sqrt{2} K_{p d_{1}} & \sqrt{2} K_{z d_{1}} \\
\sqrt{2} K_{p d_{1}} & i \sqrt{2} K_{p d_{1}} & K_{p}^{\mathrm{O}} & 0 & 0 \\
i \sqrt{2} K_{p d_{1}} & -\sqrt{2} K_{p d_{1}} & 0 & K_{p}^{\mathrm{O}} & 0 \\
i \sqrt{2} K_{z d_{1}} & \sqrt{2} K_{z d_{1}} & 0 & 0 & K_{z}^{\mathrm{O}}
\end{array}\right) .
\end{gathered}
$$

As for the $\Gamma$ point, also here the upper labels $(\mu=\mathrm{E}, \mathrm{O})$ in $K_{\alpha}^{\mu}(\mu=\mathrm{E}, \mathrm{O})$ express the symmetry of the state corresponding to the energy level $K_{\alpha}^{\mu}$ with respect to the $z \rightarrow-z$ inversion. The electronic properties of the Hamiltonian at the K point look more transparent by introducing a different "chiral" basis,

$$
\bar{\psi}_{k}^{\dagger}=\left(d_{k, 3 z^{2}-r^{2}}^{\dagger}, d_{k, L 2}^{\dagger}, d_{k, R 2}^{\dagger}, p_{k, L, S}^{\dagger}, p_{k, R, S}^{\dagger}, p_{k, z, A}^{\dagger}, d_{k, L 1}^{\dagger}, d_{k, R 1}^{\dagger}, p_{k, L, A}^{\dagger}, p_{k, R, A}^{\dagger}, p_{k, z, S}^{\dagger}\right),
$$

where $\quad d_{k, L 2}=\left(d_{k, x^{2}-y^{2}}-i d_{k, x y}\right) / \sqrt{2}, \quad d_{k, R 2}=\left(d_{k, x^{2}-y^{2}}+\right.$ $\left.i d_{k, x y}\right) / \sqrt{2}, \quad d_{k, L 1}=\left(d_{k, x z}-i d_{k, y z}\right) / \sqrt{2}, \quad d_{k, R 1}=\left(d_{k, x z}+\right.$ $\left.i d_{k, y z}\right) / \sqrt{2}, \quad p_{k, L, S}=\left(p_{k, x, S}-i p_{k, y, S}\right) / \sqrt{2}, \quad p_{k, R, S}=$ $\left(p_{k, x, S}+i p_{k, y, S}\right) / \sqrt{2}, \quad p_{k, L, A}=\left(p_{k, x, A}-i p_{k, y, A}\right) / \sqrt{2}$, $p_{k, R, A}=\left(p_{k, x, A}+i p_{k, y, A}\right) / \sqrt{2}$.

In this basis, the Hamiltonian matrix can be also divided in smaller sub-blocks (see Appendix B) as

$$
\hat{H}_{\mathrm{E}}(\mathrm{K})=\left(\begin{array}{ccc}
\hat{H}_{p d_{0}}(\mathrm{~K}) & 0 & 0 \\
0 & \hat{H}_{z d_{2}}(\mathrm{~K}) & 0 \\
0 & 0 & \hat{H}_{p d_{2}}(\mathrm{~K})
\end{array}\right)
$$

and

$$
\hat{H}_{\mathrm{O}}=\left(\begin{array}{ccc}
\hat{H}_{p d_{1}}(\mathrm{~K}) & 0 & 0 \\
0 & \hat{H}_{z d_{1}}(\mathrm{~K}) & 0 \\
0 & 0 & K_{p}^{\mathrm{O}}
\end{array}\right) .
$$

As is evident from the labels, each sub-block is also here a $2 \times 2$ dimer, apart from the term $K_{p}^{O}$ which is a $1 \times 1$ block (monomer) with pure $p_{x}, p_{y}$ character. The association between the DFT energy levels and the TB eigenstates is reported also for the $\mathrm{K}$ point in Table $\mathrm{I}$.

\section{Q point}

As discussed above, another special point determining the electronic properties of $\mathrm{MoS}_{2}$ is the Q point, halfway between the $\Gamma$ and $\mathrm{K}$ points in the Brillouin zone, where the conduction band, in the single-layer system, has a secondary minimum in addition to the absolute one at the K point. Unfortunately, not being a point of high symmetry, the TB Hamiltonian cannot be decomposed in this case in simpler smaller blocks. Each energy eigenvalue will contain thus a finite component of all the Mo and S orbitals. In particular, focusing on the secondary minimum in Q, DFT calculations give $46 \% d_{2}, 24 \% p_{x / y}$, $11 \% p_{z}$, and $9 \% d_{0}$. The orbital content of this level will play a crucial role in determining the band structure of multilayer compounds.

\section{Orbital constraints for a tight-binding model}

After having investigated in detail the orbital contents of each eigenstate at the high-symmetry points and having 
identified them with the corresponding DFT energy levels, we can now employ such analysis to assess the basic conditions that a TB model must fulfill and to elucidate the physical consequences.

A first interesting issue is about the minimum number of orbitals needed to be taken into account in a TB model for a robust description of the electronic properties of these materials. A proper answer to such an issue is, of course, different if referred to single-layer or multilayer compounds. For the moment we focus only on the single-layer case but we emphasize the way the relevant features that will be needed to take into account in multilayer systems.

In a single-layer case, focusing only on the band edges determined by the states (A) and (B) at the $\mathrm{K}$ point, we can identify them with the eigenstates $E_{p d_{0},+}(\mathrm{K}), E_{p d_{2},+}(\mathrm{K})$, respectively, with a dominant Mo $4 d$ character and a marginal $\mathrm{S} p_{x / y}$ component, as we show below. It is thus tempting to define a reduced three-band TB model, keeping only the Mo $4 d_{3 z^{2}-r^{2}}, 4 d_{x^{2}-y^{2}}, 4 d_{x y}$ orbitals with dominant character and disregarding the $\mathrm{S} p_{x}, p_{y}$ orbitals, with a small marginal weight. A similar phenomenological model was proposed in Ref. 25. However, the full microscopic description here exposed makes it possible to point out the inconsistency of such a model. This can be shown by looking at Eq. (18). The band gap at $\mathrm{K}$ in the full TB model including $\mathrm{S} p_{x}, p_{y}$ orbitals is determined by the upper eigenstate of $\hat{H}_{p d_{0}}$,

$$
E_{p d_{0},+}(\mathrm{K})=\frac{K_{0}+K_{p}^{\mathrm{E}}}{2}+\sqrt{\left(\frac{K_{0}-K_{p}^{\mathrm{E}}}{2}\right)^{2}+4 K_{p d_{0}}^{2}},
$$

and the upper eigenstate of $\hat{H}_{p d_{2}}$,

$$
E_{p d_{2},+}(\mathrm{K})=\frac{K_{2}+K_{p}^{\mathrm{E}}}{2}+\sqrt{\left(\frac{K_{2}-K_{p}^{\mathrm{E}}}{2}\right)^{2}+8 K_{p d_{2}}^{2}},
$$

both with main Mo $4 d$ character, while the eigenstate

$$
E_{z d_{2},+}(\mathrm{K})=\frac{K_{2}+K_{z}^{\mathrm{E}}}{2}+\sqrt{\left(\frac{K_{2}-K_{z}^{\mathrm{E}}}{2}\right)^{2}+4 K_{z d_{2}}^{2}},
$$

also with dominant Mo $4 d$ character, but belonging to the block $\hat{H}_{z d_{2}}$, lies at higher energy (see Table I). The three-band model retaining only the $d_{0}, d_{2}$ orbitals is equivalent to switching off the hybridization terms $K_{p d_{0}}, K_{p d_{2}}, K_{z d_{2}}$, ruled by $V_{p d \sigma}$, $V_{p d \pi}$, so that $E_{p d_{0},+}(\mathrm{K})=K_{0}, E_{p d_{2},+}(\mathrm{K})=E_{z d_{2},+}(\mathrm{K})=K_{2}$. In this context the level $E_{z d_{2},+}(\mathrm{K})$ becomes degenerate with $E_{p d_{2},+}(\mathrm{K})$. This degeneracy is not accidental but it reflects the fact that the elementary excitations of the $d_{2}$ states, in this simplified model, are described by a Dirac spectrum, as sketched in Fig. 4. As a consequence, no direct gap can be possibly established in this framework. It is worth mentioning that a spin-orbit coupling can certainly split the Dirac cone to produce a direct gap at the $\mathrm{K}$ point, but it would not explain in any case the direct gap observed in the DFT calculations without spin-orbit coupling.

We should also mention that, in the same reduced threeband model keeping only the $d_{0}$ and $d_{2}$ Mo orbitals, the secondary maximum $(\mathrm{C})$ of the valence band would have a pure $d_{0}$ orbital character. As we see in the discussion concerning the multilayer samples, this would have important consequences on the construction of a proper TB model.

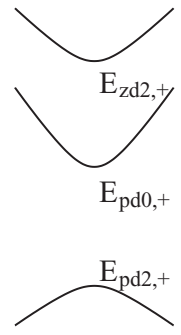

(a)

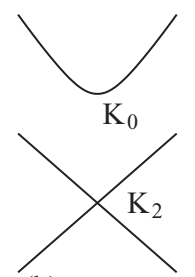

(b)
$\mathrm{K}$

K

FIG. 4. Schematic band structure close to the $\mathrm{K}$ point for the valence and conduction bands: (a) including $\mathrm{S} p_{x}, p_{y}$ orbitals; (b) omitting $\mathrm{S} p_{x}, p_{y}$ orbitals.

A final consideration concerns the orbital character of the valence band edge, $E_{p d_{2},+}(\mathrm{K})$. This state is associated with the third $2 \times 2$ block of (18) and it results from the hybridization of the chiral state $d_{k, R 2}=\left(d_{k, x^{2}-y^{2}}+i d_{k, x y}\right) / \sqrt{2}$ of the Mo $d$ orbitals with the chiral state $p_{k, R, S}=\left(p_{k, x, S}+\right.$ $\left.i p_{k, y, S}\right) / \sqrt{2}$ of the $\mathrm{S} p$ orbitals. The role of the chirality associated with the $d$ orbitals, in the presence of a finite spin-orbit coupling, has been discussed in detail in relation with spin/valley selective probes. ${ }^{21-27}$ What results from a careful TB description is that such $d$-orbital chirality is indeed strongly associated with a corresponding chirality associated with the $\mathrm{S} p$ orbitals. The possibility of such a correlation, dictated by group theory, was pointed out in Ref. 28.

A similar feature is found for the conduction band edge, $E_{p d_{0},+}(\mathrm{K})$. So far, this state has been assumed to be mainly characterized by the $d_{3 z^{2}-r^{2}}$ and hence without an orbital moment. However, as we can see, this is true only for the Mo $d$ part, whereas the $\mathrm{S} p$ component does contain a finite chiral moment. On the other hand, the spin-orbit associated with the $\mathrm{S}$ atoms as well as with other chalcogenides (e.g., Se) is quite small, and taking into account also the small orbital $\mathrm{S}$ weight, the possibility of a direct probe of such orbital moment is still to be explored.

\section{BULK SYSTEM}

In the previous section we examined in detail the content of the orbital character in the main high-symmetry points of the Brillouin zone of the single-layer $\mathrm{MoS}_{2}$, to provide theoretical constraints on the construction of a suitable TB model. Focusing on the low-energy excitations close to the direct gap at the $\mathrm{K}$ point, we have seen that a proper model must take into account at least the three Mo orbitals $d_{3 z^{2}-r^{2}}$, $d_{x^{2}-y^{2}}, d_{x y}$ and the two S orbitals $p_{x}, p_{y}$. On the other hand, our wider aim is to introduce a TB model for the single layer that would be the basic ingredient for a TB model in multilayer systems, simply adding the interlayer coupling.

For the sake of simplicity we focus here on the bulk $2 \mathrm{H}$ $\mathrm{MoS}_{2}$ structure as a representative case that contains already all the ingredients of the physics of multilayer compounds. The band structure for the bulk compound is shown in Fig. 3. As it is known, the secondary maximum (C) of valence band at the $\Gamma$ point is shifted to higher energies in multilayer 
systems with respect to the single-layer case, becoming the valence band maximum. At the same time also the secondary minimum (D) of the conduction band, roughly at the $\mathrm{Q}$ point, is lowered in energy, becoming the conduction band minimum. All these changes result in a transition between a direct gap material in single-layer compounds and indirect gap systems in the multilayer case. Although such an intriguing feature has been discussed extensively and experimentally observed, the underlying mechanism has not been so far elucidated. We show here that such topological transition of the band edges can be naturally explained within the context of a TB model as a result of an orbital selective (and hence momentum dependent) band splitting induced by the interlayer hopping.

The orbital content of the bulk band structure along the same high-symmetry lines as in the single-layer case is shown in Fig. 3. We focus first on the K point, where the single-layer system has a direct gap. We note that the direct gap at $\mathrm{K}$ is hardly affected. The interlayer coupling produces just a very tiny splitting of the valence band edge $E_{p d_{2},+}(\mathrm{K})$, while the conduction band edge $E_{p d_{0},+}(\mathrm{K})$ at $\mathrm{K}$ becomes doubly degenerate.

Things are radically different at the $\Gamma$ point. The analysis of the orbital weight $d_{3 z^{2}-r^{2}}$ in Fig. 3 shows indeed that there is a sizable splitting of the $E_{z d_{0},+}(\Gamma)$ level, of the order of $1 \mathrm{eV}$. A bit more difficult to discern, because of the multiorbital component, but still visible, is the splitting of the secondary minimum (D) of the conduction band in $\mathrm{Q}$. This is detected most clearly in Fig. 3 by looking at the $d_{2}$ and $d_{0}$ characters, which belong uniquely to the E block. One can thus estimate from DFT a splitting of this level at the Q point of $\sim 1.36 \mathrm{eV}$.

We are now going to see that all these features are consistent with a TB construction where the interlayer hopping acts as an additional parameter with respect to the single-layer TB model. From the TB point of view, it is clear that the main processes to be included are the interlayer hoppings between the external $\mathrm{S}$ planes of each $\mathrm{MoS}_{2}$ block. This shows once more the importance of including the $\mathrm{S} p$ orbital in a reliable TB model. Moreover, for geometric reasons, one could expect that the interlayer hopping between the $p_{z}$ orbitals, pointing directly out of plane, would be dominant with respect to the interlayer hopping between $p_{x}$ and $p_{y}$. This qualitative argument is supported by the DFT results, which indeed report a big splitting of the $E_{z d_{0},+}(\Gamma)$ level at the $\Gamma$ point, with $27 \%$ of the $p_{z}$ component, but almost no splitting of the degenerate $E_{p d_{2},+}(\Gamma)$ at $\sim 2 \mathrm{eV}$, with $68 \%$ of the component of $p_{x}, p_{y}$.

We can quantify this situation within the TB description by including explicitly the interlayer hopping between the $p$ orbitals of the $\mathrm{S}$ atoms in the outer planes of each $\mathrm{MoS}_{2}$ layer, with interatomic distance $d=3.49 \AA$ (see Fig. 1). These processes will be parametrized in terms of the interlayer Slater-Koster ligands $U_{p p \sigma}, U_{p p \pi}$. The Hilbert space is now determined by a 22 -fold vector, defined as

$$
\tilde{\Phi}_{k}^{\dagger}=\left(\tilde{\phi}_{k, 1}^{\dagger}, \tilde{\phi}_{k, 2}^{\dagger}\right)
$$

where $\tilde{\phi}_{k, 1}^{\dagger}$ represents the basis (9) for the layer 1 , and $\tilde{\phi}_{k, 2}^{\dagger}$ is the same quantity for layer 2 . The corresponding Hamiltonian, in the absence of interlayer hopping, would read

$$
\hat{H}_{\text {bulk }}=\left(\begin{array}{cc}
\hat{H}_{1} & \hat{0} \\
\hat{0} & \hat{H}_{2}
\end{array}\right),
$$

where $\hat{H}_{1}, \hat{H}_{2}$ refer to the intralayer Hamiltonian for the layers 1 and 2 , respectively.

Note that the Hamiltonian of layer 2 in the $2 \mathrm{H}-\mathrm{MoS}_{2}$ structure is different with respect to the one of layer 1. From a direct inspection we can see that the elements $H_{2, \alpha, \beta}(\xi, \eta)$ of layer 2 are related to the corresponding elements of layer 1 as

$$
H_{2, \alpha, \beta}(\xi, \eta)=P_{\alpha} P_{\beta} H_{1, \alpha, \beta}(\xi,-\eta),
$$

where $\xi=k_{x} a / 2, \eta=\sqrt{3} k_{y} a / 2$, and $P_{\alpha}=1$ if the orbital $\alpha$ has even symmetry for $y \rightarrow-y$, and $P_{\alpha}=-1$ if it has odd symmetry. We note that both effects can be readsorbed in a different redefinition of the orbital basis so that the eigenvalues of $\hat{H}_{2}$ are, of course, the same as the eigenvalues of $\hat{H}_{1}$.

Taking into account the interlayer S-S hopping terms, we can write

$$
\hat{H}_{\text {bulk }}=\left(\begin{array}{cc}
\hat{H}_{1} & \hat{H}_{\perp} \\
\hat{H}_{\perp}^{\dagger} & \hat{H}_{2}
\end{array}\right),
$$

where $\hat{H}_{\perp}$ is here the interlayer hopping Hamiltonian, namely,

$$
\hat{H}_{\perp}=\left(\begin{array}{cc}
\hat{I}_{\mathrm{E}} \cos \zeta & \hat{I}_{\mathrm{EO}} \sin \zeta \\
-\hat{I}_{\mathrm{EO}}^{\mathrm{T}} \sin \zeta & \hat{I}_{\mathrm{O}} \cos \zeta
\end{array}\right),
$$

where $\zeta=k_{z} c / 2$ and

$$
\begin{gathered}
\hat{I}_{\mathrm{E}}=\left(\begin{array}{cc}
\hat{0}_{3 \times 3} & \hat{0}_{3 \times 3} \\
\hat{0}_{3 \times 3} & \hat{I}
\end{array}\right), \\
\hat{I}_{\mathrm{O}}=\left(\begin{array}{cc}
\hat{0}_{2 \times 2} & \hat{0}_{2 \times 3} \\
\hat{0}_{3 \times 2} & \hat{I}
\end{array}\right), \\
\hat{I}_{\mathrm{EO}}=\left(\begin{array}{cc}
\hat{0}_{3 \times 2} & \hat{0}_{3 \times 3} \\
\hat{0}_{3 \times 2} & i \hat{I}
\end{array}\right), \\
\hat{I}=\left(\begin{array}{ccc}
I_{x / x} & I_{x / y} & I_{x / z} \\
I_{x / y} & I_{y / y} & I_{y / z} \\
I_{x / z} & I_{y / z} & I_{z / z}
\end{array}\right) .
\end{gathered}
$$

The analytical expression of the elements $I_{\alpha / \beta}$ as functions of the Slater-Koster interlayer parameters $U_{p p \sigma}, U_{p p \pi}$ is provided in Appendix A. Note that, in the presence of interlayer hopping in the bulk $\mathrm{MoS}_{2}$, we cannot divide anymore, for generic momentum $\mathbf{k}$, the $22 \times 22$ Hamiltonian in smaller blocks with even and odd symmetry with respect to the change $z \rightarrow-z$. The analysis is, however, simplified at specific high-symmetry points of the Brillouin zone. In particular, for $k_{z}=0(\zeta=0)$, we can easily see from (27) that the block $12 \times 12(6 \times 6+6 \times 6)$ with even symmetry and the block $10 \times 10(5 \times 5+5 \times 5)$ with odd symmetry are still decoupled.

Exploiting this feature, we can now give a closer look at the high-symmetry points.

\section{A. $\Gamma$ point}

In Sec. III we have seen that at the $\Gamma$ point the Hamiltonian can be decomposed in $2 \times 2$ blocks. Particularly important 
here is the block $H_{z d_{0}}$ whose upper eigenvalue $E_{z d_{0},+}(\Gamma)$, with main orbital character $d_{3 z^{2}-r^{2}}$ and a small $p_{z}$ component, represents the secondary maximum $(\mathrm{C})$ of the valence band. A first important property to be stressed in bulk systems is that, within this $(\mathrm{Mo} 4 d)+(\mathrm{S} 3 p) \mathrm{TB}$ model, the interlayer coupling at the $\Gamma$ point does not mix any additional orbital character. This can be seen by noticing that the interlayer matrix $\hat{I}$ is diagonal at the $\Gamma$ point. Focusing on the $E_{z d_{0}}(\Gamma)$ levels, we can write thus a $4 \times 4$ reduced Hamiltonian (see Appendix B),

$$
\hat{H}_{z d_{0}}=\left(\begin{array}{cccc}
\Gamma_{0} & \sqrt{2} \Gamma_{z d_{0}} & 0 & 0 \\
\sqrt{2} \Gamma_{z d_{0}} & \Gamma_{z}^{\mathrm{E}} & 0 & \Gamma_{z z} \\
0 & 0 & \Gamma_{0} & \sqrt{2} \Gamma_{z d_{0}} \\
0 & \Gamma_{z z} & \sqrt{2} \Gamma_{z d_{0}} & \Gamma_{z}^{\mathrm{E}}
\end{array}\right),
$$

where $\Gamma_{z z}$ represents the interlayer hopping mediated by $U_{p p \sigma}$, $U_{p p \pi}$ between $p_{z}$ orbitals belonging to the outer $\mathrm{S}$ planes on different layers. Equation (32) is important since it shows the correctness of the qualitative idea that each level in the bulk system is just split by the interlayer hopping. In particular, under the reasonable hypothesis that the interlayer hopping is much smaller than intralayer processes, denoting $E_{z d_{0},+a}(\Gamma)$, $E_{z d_{0},+b}(\Gamma)$ the two eigenvalues with primary $d_{0}$ components, we get

$$
\begin{aligned}
\Delta E_{z d_{0},+}(\Gamma) & =E_{z d_{0},+a}(\Gamma)-E_{z d_{0},+b}(\Gamma) \\
& \approx \Gamma_{z z}\left[\frac{\Gamma_{0}-\Gamma_{z}^{\mathrm{E}}}{2 \sqrt{\left(\frac{\Gamma_{0}-\Gamma_{z}^{\mathrm{E}}}{2}\right)^{2}+2 \Gamma_{z d_{0}}}}-1\right] \\
& =\Gamma_{z z}\left[\frac{\Gamma_{0}-\Gamma_{z}^{\mathrm{E}}}{E_{z d_{0},+}(\Gamma)-E_{z d_{0},-}(\Gamma)}-1\right] .
\end{aligned}
$$

A similar situation is found for the other $2 \times 2$ blocks, $\hat{H}_{p d_{2}}(\Gamma), \hat{H}_{p d_{1}}(\Gamma)$, and the $1 \times 1$ block $\hat{H}_{z}(\Gamma)$. Most important, tracking the DFT levels by means of their orbital content, we can note that both levels $E_{z d 0,+}(\Gamma)$ and $E_{z d 0,-}(\Gamma)$ undergo a quite large splitting $\approx 1.2 \mathrm{eV}$, and the level $E_{z}(\Gamma)$ a splitting $\approx 2.6 \mathrm{eV}$, whereas the levels $\hat{H}_{p d_{2}}(\Gamma), \hat{H}_{p d_{1}}(\Gamma)$ are almost unsplit. This observation strongly suggests that, as expected, the interlayer hopping between $p_{x}$ and $p_{y}$ orbitals is much less effective than the interlayer hopping between $p_{z}$ orbitals.

A similar conclusion can be drawn from the investigation of the energy levels at the $\mathrm{K}$ point, although the analysis is a bit more involved.

\section{B. K point}

The properties of the bulk system at the K point are dictated by the structure of the interlayer matrix $\hat{I}$ which, in the basis defined in Eq. (23), at the $\mathrm{K}$ point reads

$$
\hat{I}_{66}(\mathrm{~K})=\left(\begin{array}{ccc}
K_{p p} & i K_{p p} & i K_{p z} \\
i K_{p p} & -K_{p p} & K_{p z} \\
i K_{p z} & K_{p z} & 0
\end{array}\right) .
$$

As discussed in detail in Appendix B, the electronic structure is made more transparent by using an appropriate chiral basis, which is a direct generalization of the one for the single layer. We can thus write the even and odd parts of the resulting Hamiltonian in the form

$$
\begin{aligned}
\hat{H}_{\mathrm{E}}(\mathrm{K}) & =\left(\begin{array}{ccc}
\hat{H}_{p z d_{02}}(\mathrm{~K}) & 0 & 0 \\
0 & \hat{H}_{p z d_{02}}(\mathrm{~K}) & 0 \\
0 & 0 & \hat{H}_{p d_{2}, \mathrm{E}}(\mathrm{K})
\end{array}\right), \\
\hat{H}_{\mathrm{O}}(\mathrm{K}) & =\left(\begin{array}{ccc}
\hat{H}_{p z d_{1}}(\mathrm{~K}) & 0 & 0 \\
0 & \hat{H}_{p z d_{1}}(\mathrm{~K}) & 0 \\
0 & 0 & \hat{H}_{p d_{1}, \mathrm{O}}(\mathrm{K})
\end{array}\right),
\end{aligned}
$$

where

$$
\hat{H}_{p z d_{02}}(\mathrm{~K})=\left(\begin{array}{cccc}
K_{0} & -2 i K_{p d_{0}} & 0 & 0 \\
2 i K_{p d_{0}} & K_{p}^{E} & 0 & i \sqrt{2} K_{p z} \\
0 & 0 & K_{2} & 2 K_{z d_{2}} \\
0 & -i \sqrt{2} K_{p z} & 2 K_{z d_{2}} & K_{z}^{\mathrm{E}}
\end{array}\right),
$$

$$
\begin{aligned}
& \hat{H}_{p d_{2}, \mathrm{E}}(\mathrm{K}) \\
& =\left(\begin{array}{cccc}
K_{2} & i \sqrt{8} K_{p d_{2}} & 0 & 0 \\
-i \sqrt{8} K_{p d_{2}} & K_{p}^{E} & 0 & 2 K_{p p} \\
0 & 0 & K_{2} & i \sqrt{8} K_{p d_{2}} \\
0 & 2 K_{p p} & -i \sqrt{8} K_{p d_{2}} & K_{p}^{\mathrm{E}}
\end{array}\right), \\
& \hat{H}_{p z d_{1}}(\mathrm{~K})=\left(\begin{array}{cccc}
K_{1} & -2 i K_{z d_{1}} & 0 \\
2 i K_{z d_{1}} & K_{z}^{\mathrm{O}} & 0 \\
0 & 0 & K_{p}^{\mathrm{O}}
\end{array}\right), \\
& \hat{H}_{p d_{1}, \mathrm{O}}(\mathrm{K})=\left(\begin{array}{cccc}
K_{1} \\
\sqrt{8} K_{p d_{1}} & K_{p}^{\mathrm{O}} K_{p d_{1}} & 0 & \\
0 & 0 & 0 & 2 K_{p p} \\
0 & 2 K_{p p} & \sqrt{8} K_{p d_{1}} & K_{p}^{\mathrm{O}}
\end{array}\right) .
\end{aligned}
$$

We can notice that Eq. (38) has the same structure as (32), with two $2 \times 2$ degenerate sub-blocks hybridized by a nondiagonal element $\left(K_{p p}\right.$ in this case). This results in a splitting of the single-layer levels $\quad E_{p d_{2},+}(\mathrm{K}) \rightarrow E_{p d_{2},+a}(\mathrm{~K}), E_{p d_{2},+b}(\mathrm{~K}), \quad E_{p d_{2},-}(\mathrm{K}) \rightarrow$ $E_{p d_{2},-a}(\mathrm{~K}), E_{p d_{2},-b}(\mathrm{~K})$. The two levels $E_{p d_{2},+a}(\mathrm{~K})$, $E_{p d_{2},+b}(\mathrm{~K})$, by looking at their orbital character, can be identified in DFT results in the small splitting of the (B) $E_{p d_{2},+}(\mathrm{K})$ level, confirming once more the smallness of the interlayer $p_{x / y}-p_{x / y}$ hopping.

Less straightforward is the case of the $4 \times 4$ block $\hat{H}_{p z d_{02}}(\mathrm{~K})$, where the hybridization term $\sqrt{2} K_{p z}$ mixes two different $2 \times 2$ sub-blocks, $\hat{H}_{p d_{0}}$ and $\hat{H}_{z d_{2}}$. In this case, a mixing of the orbital character will result. We note, however, that the block $\hat{H}_{p z d_{02}}(\mathrm{~K})$ appears twice in (35), so that each energy level will be doubly degenerate, in particular the minimum (A) of the conduction band at K. Note, however, that the negligible shift of such energy level in the DFT calculations with respect to the single-layer case is an indication that also the interlayer hopping element $K_{p z}$, between $p_{z}$ on one layer and $p_{x}, p_{y}$ on the other one, is negligible. 


\section{Q point}

An analytical insight into the electronic structure at the Q point was not available in single-layer systems and it would be thus even more complicated in the bulk case. A few important considerations, concerning the minimum (D), can, however, be drawn from the DFT results. In particular, we note that in the single-layer case this energy level had a nonvanishing $p_{z}$ component. As we have seen above, the interlayer hopping between $p_{z}$ orbitals appears to be dominant with respect to the interlayer hopping between $p_{x / y}$ and $p_{x / y}$ and with respect to the mixed interlayer hopping $p_{z}-p_{x / y}$. We can thus expect a finite sizable splitting of the (D) level, containing a finite $p_{z}$ component, with respect to the negligible energy shift of $E_{p d_{0},+}$ (A), which depends on the mixed interlayer process $K_{p z}$.

\section{MOMENTUM/ORBITAL SELECTIVE SPLITTING AND COMPARISON WITH DFT DATA}

In the previous section we elucidated, using a TB model, the orbital character of the band structure of $\mathrm{MoS}_{2}$ on the main high-symmetry points of the Brillouin zone. We have shown how a reliable minimal model for the single-layer case needs to take into account at least the $p_{x}, p_{y}$ orbitals of the $\mathrm{S}$ atoms in addition to the $4 d$ orbitals of Mo. A careful inspection of the electronic structure shows also that the band edges at the $\mathrm{K}$ point defining the direct band gap in the single-layer case are characterized not only by a chiral order of the $d$ Mo orbitals, as experimentally observed, but also by a chiral order of the minor component of the $p_{x / y} \mathrm{~S}$ orbitals.

An important role is also played by the $p_{z}$ orbitals of the $\mathrm{S}$ atoms. In single-layer systems, the $p_{z}$ orbital character is particularly relevant in the $(C)$ state, characterizing a secondary maximum in the valence band at the $\Gamma$ point, and in the (D) state, which instead provides a secondary minimum in the conduction band at the $\mathrm{Q}$ point.

The $p_{z}$ component becomes crucial in multilayer compounds where a comparison with DFT results shows that the interlayer coupling is mainly driven by the $p_{z}-p_{z}$ hopping, whereas $p_{x / y}-p_{x / y}, p_{z}-p_{x / y}$ are negligible. This results in an orbital-selective and momentum-dependent interlayer splitting of the energy levels, being larger for the (C) and (D) states and negligible for (A) and (B). This splitting is thus the fundamental mechanism responsible for the transition from a direct (A)-(B) gap in single-layer compounds to an indirect (C)-(D) gap in multilayer systems. Controlling these processes is therefore of the highest importance for electronic applications. Note that such direct/indirect gap switch is discussed here in terms of the number of layers. On the other hand, the microscopical identification of such a mechanism, which is essentially driven by the interlayer coupling, makes it possible to understand on the physical ground the high sensitivity to pressure/strain effects, as well as to the temperature, via the lattice expansion.

Finally, in order to show at a quantitative level how the orbital content determines the evolution of the electronic structure from single-layer to multilayer compounds, we have performed a fitting procedure to determine the TB parameters that best reproduce the DFT bands within the model defined here. The task was divided into two steps: (i) We first focus on the single-layer case to determine the relevant Slater-Koster intralayer parameters in this case; (ii) afterwards, keeping fixed the intralayer parameters, we determine the interlayer parameters. To this purpose we employ a simplex method ${ }^{105}$ to minimize a weighted mean square error $f_{\mathrm{wMSE}}$ between the TB and DFT band energies, defined as

$$
f_{\mathrm{wMSE}}=\sum_{\mathbf{k}, i} w_{i}(\mathbf{k})\left[\epsilon_{i}^{\mathrm{TB}}(\mathbf{k})-\epsilon_{i}^{\mathrm{DFT}}(\mathbf{k})\right]^{2},
$$

where $\epsilon_{i}^{\mathrm{DFT}}(\mathbf{k})$ is the dispersion on the $i$ th band of the 11-band block under consideration, $\epsilon_{i}^{\mathrm{TB}}(\mathbf{k})$ is the corresponding $\mathrm{TB}$ description, and $w_{i}(\mathbf{k})$ is a band/momentum resolved weight which can be used to improve fitting over particular $\mathbf{k}$ regions or over selected bands. In spite of many efforts, we could not find a reliable fit for the whole electronic structure including the seven valence bands and the four lowest conduction bands. ${ }^{106}$ As our analysis and our main objective concerns the description of the valence and conduction bands that define the band gap of these systems, we focus on finding a set of parameters that describe properly these bands. Since both the lowest conduction and highest valence band belong to the electronic states with even $z \rightarrow-z$ symmetry, the fit was performed in the $6 \times 6$ orbital space defined by this symmetry. In addition, due to the degeneracy at the $\Gamma$ point and to the band crossing along the $\Gamma-\mathrm{M}$ direction, the two conduction bands with even symmetry for $z \rightarrow-z$ were considered in the fit. Additionally, we give a larger weight to the (A)-(D) band edges in order to obtain a better description of the most important features of the band structure.

Our best fit for the single-layer case is shown in the top panel of Fig. 5 (where only the TB bands with even symmetry $z \rightarrow-z$ are shown), compared with the DFT bands, and the

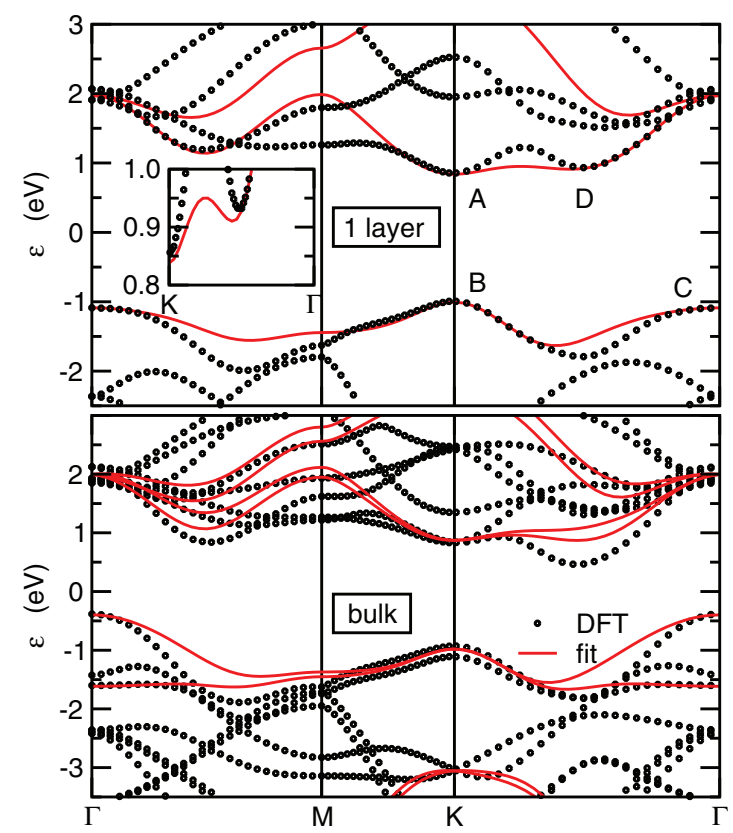

FIG. 5. (Color online) Comparison between the DFT band structure (black dots) and the best fit TB model (red solid lines) for single-layer (top panel) and bulk $\mathrm{MoS}_{2}$ (bottom panel). (Inset in the top panel) Magnification of the conduction bands along the $\mathrm{K}-\Gamma$ direction in the single-layer case. 
TABLE II. Tight-binding parameters for single-layer $\operatorname{MoS}_{2}\left(\Delta_{\alpha}\right.$, $\left.V_{\alpha}\right)$ as obtained by fitting the low-energy conduction and valence bands. Also shown are the interlayer hopping parameters $U_{\alpha}$ relevant for bulk $\mathrm{MoS}_{2}$. All hopping terms $V_{\alpha}, U_{\alpha}$ and crystal fields $\Delta_{\alpha}$ are in units of eV.

\begin{tabular}{lcr}
\hline \hline Crystal fields & $\Delta_{0}$ & -1.512 \\
& $\Delta_{1}$ & \\
& $\Delta_{2}$ & -3.025 \\
& $\Delta_{p}$ & -1.276 \\
& $\Delta_{z}$ & -8.236 \\
Intralayer Mo-S & $V_{p d \sigma}$ & -2.619 \\
& $V_{p d \pi}$ & -1.396 \\
Intralayer Mo-Mo & $V_{d d \sigma}$ & -0.933 \\
& $V_{d d \pi}$ & -0.478 \\
Intralayer S-S & $V_{d d \delta}$ & -0.442 \\
& $V_{p p \sigma}$ & 0.696 \\
Interlayer S-S & $V_{p p \pi}$ & 0.278 \\
& $U_{p p \sigma}$ & -0.774 \\
& $U_{p p \pi}$ & 0.123 \\
\hline \hline
\end{tabular}

corresponding TB parameters are listed in Table II. Note that, due to the restriction of our fitting procedure to only some bands belonging to the block with even symmetry, the atomic crystal field $\Delta_{1}$ for the Mo orbitals $d_{x z}, d_{y z}$ (not involved in the fitting procedure) results undetermined. The fit reported in Fig. 5 agrees in a qualitative way with the DFT results, showing, in particular, a direct gap at the $K$ point [(A) and (B) band edges] and secondary band edges for the valence and conduction bands lying at the $\Gamma(\mathrm{C})$ and the $\mathrm{Q}$ points (D), respectively. A magnification of the valence band along the $\mathrm{K}-\Gamma$ direction is shown in the inset of the top panel of Fig. 5. The presence of such secondary minimum at the $Q$ point in the TB modeling, although less deep than in DFT calculations, is here very important because it represents a trademark of the correct orbital character.

Turning now to the bulk system, the further step of determining the interlayer hopping parameters $U_{p p \sigma}, U_{p p \pi}$ is facilitated by the strong indication, from the DFT analysis, of a dominant role of the interlayer hopping between the $p_{z}$ orbitals and a negligible role of the interlayer hopping between the $p_{x / y}$ orbitals. Focusing on the $\Gamma$ point, these two different hopping processes are parametrized in terms of the corresponding interlayer parameters $\Gamma_{z z}$ and $\Gamma_{p p}$, as discussed in Appendix B. We can thus approximate $\Gamma_{p p}=0$, providing a constraint between $U_{p p \sigma}$ and $U_{p p \pi}$ and leaving thus only one effective independent fitting parameter: $\Gamma_{z z}$. We determine it, and hence $U_{p p \sigma}$ and $U_{p p \pi}$, by fixing the effective splitting of the $E_{z d_{0},+}(\Gamma)$ level as in the DFT data. The values of $U_{p p \sigma}$ and $U_{p p \pi}$ found in this way are also reported in Table II, and the resulting band structure is shown in the bottom panel of Fig. 5, where only the TB bands with even symmetry $z \rightarrow-z$ are shown. We stress that the intralayer hoppings are here taken from the fitting of the single-layer case. The agreement between the DFT and the TB bands is also qualitatively good in this case. In particular, we would like to stress the momentum/orbital selective interlayer splitting of the bands, which is mainly concentrated at the $\Gamma$ point for the valence band and at the $Q$ point for the conduction band. This yields to the crucial transition between a direct gap in single-layer $\mathrm{MoS}_{2}$, located at the $\mathrm{K}$ point, to an indirect gap $\Gamma-\mathrm{Q}$ in multilayer systems.

On more quantitative grounds, we can see that, while the interlayer splitting of the conduction level $E_{z d_{0},+}(\Gamma)$ is easily reproduced, the corresponding splitting of the conduction band at the $\mathrm{Q}$ point is somewhat underestimated in the TB model $(0.20 \mathrm{eV})$ as compared to the DFT data $(1.36 \mathrm{eV}){ }^{107}$ This discrepancy is probably due to the underestimation, in the TB model, of the $p_{z}$ character of the conduction band at the $\mathrm{Q}$ point. As a matter of fact, the set of TB parameters reported in Table II gives at the $\mathrm{Q}$ point of the conduction band, for the single-layer case, only a $3.8 \%$ of $p_{z}$ orbital character, in comparison with the $11 \%$ found by the DFT calculations. It should be kept in mind, however, that the optimization of the TB fitting parameters in such a large phase space (12 free parameters) is a quite complex and not unambiguous procedure, and other solutions are possible. A more refined numerical search in the optimization of the TB parameters, using global minimization techniques, might result in better comparison with the DFT results and further work along this line should be of great interest.

\section{CONCLUSIONS}

In this paper we have provided an analytic and reliable description of the electronic properties of single-layer and multilayer semiconducting transition-metal dichalcogenides in terms of a suitable TB model. We have shown that the band structure of the multilayer compounds can be generated from the TB model for the single-layer system by adding the few relevant interlayer hopping terms. The microscopic mechanism for the transition between a direct-gap to an indirect-gap from single-layer to multilayer compounds is thus explained in terms of a momentum/orbital selective interlayer band splitting, where the orbital $p_{z}$ component of the $\mathrm{S}$ atoms plays a central role. The present work provides a suitable basis for the inclusion of many-body effects within the context of QFT and for the analysis of local strain effects related to the modulation of the Mo-S, Mo-Mo, and S-S ligands.

\section{ACKNOWLEDGMENTS}

F.G. acknowledges financial support from MINECO, Spain, through Grant No. FIS2011-23713, and the European Union, through Grant No. 290846. R.R. acknowledges financial support from the Juan de la Cierva Program (MINECO, Spain). E.C. acknowledge support from the European FP7 Marie Curie Project No. PIEF-GA-2009-251904. J.A.S.-G. and P.O. acknowledge support from Spanish MINECO (Grants No. FIS2009-12721-C04-01, No. FIS2012-37549-C05-02, and No. CSD2007-00050). J.A.S.-G. was supported by an FPI Fellowship from MINECO.

\section{APPENDIX A: TIGHT-BINDING HAMILTONIAN ELEMENTS}

In this appendix we provide an analytical expression, in terms of the Slater-Koster parameters, for the several intralayer and interlayer matrix elements that appear in the Hamiltonian of the TB model. Following Ref. 104, it is 
convenient to introduce few quantities that account for the moment dispersion within the Brillouin zone, as functions of the reduced momentum variables $\xi=k_{x} a / 2, \eta=\sqrt{3} k_{y} a / 2$.

We define thus

$$
\begin{aligned}
& C_{1}(\xi, \eta)= 2 \cos (\xi) \cos (\eta / 3)+\cos (2 \eta / 3) \\
&+i[2 \cos (\xi) \sin (\eta / 3)-\sin (2 \eta / 3)], \\
& C_{2}(\xi, \eta)= \cos (\xi) \cos (\eta / 3)-\cos (2 \eta / 3) \\
&+i[\cos (\xi) \sin (\eta / 3)+\sin (2 \eta / 3)], \\
& C_{3}(\xi, \eta)= \cos (\xi) \cos (\eta / 3)+2 \cos (2 \eta / 3) \\
&+i[\cos (\xi) \sin (\eta / 3)-2 \sin (2 \eta / 3)], \\
& d_{1}(\xi, \eta)=\sin (\eta / 3)-i \cos (\eta / 3), \\
& l_{1}(\xi, \eta)=\cos (2 \xi)+2 \cos (\xi) \cos (\eta), \\
& l_{2}(\xi, \eta)=\cos (2 \xi)-\cos (\xi) \cos (\eta), \\
& l_{3}(\xi, \eta)=2 \cos (2 \xi)+\cos (\xi) \cos (\eta) .
\end{aligned}
$$

\section{Intralayer hopping terms}

Following Ref. 104, the intralayer hopping terms $H_{\alpha, \beta}$ appearing in Eqs. (4)-(8) can be written as

$$
\begin{aligned}
H_{x / x}(\xi, \eta) & =\Delta_{p}+E_{15} l_{3}(\xi, \eta)+3 E_{16} \cos (\xi) \cos (\eta) \\
H_{y / y}(\xi, \eta) & =\Delta_{p}+E_{16} l_{3}(\xi, \eta)+3 E_{15} \cos (\xi) \cos (\eta) \\
H_{z / z}(\xi, \eta) & =\Delta_{z}+2 E_{16} l_{1}(\xi, \eta) \\
H_{z^{2} / z^{2}}(\xi, \eta) & =\Delta_{0}+2 E_{9} l_{1}(\xi, \eta) \\
H_{x^{2} / x^{2}}(\xi, \eta) & =\Delta_{2}+E_{11} l_{3}(\xi, \eta)+3 E_{12} \cos (\xi) \cos (\eta) \\
H_{x y / x y}(\xi, \eta) & =\Delta_{2}+E_{12} l_{3}(\xi, \eta)+3 E_{11} \cos (\xi) \cos (\eta) \\
H_{x z / x z}(\xi, \eta) & =\Delta_{1}+E_{13} l_{3}(\xi, \eta)+3 E_{14} \cos (\xi) \cos (\eta) \\
H_{y z / y z}(\xi, \eta) & =\Delta_{1}+E_{14} l_{3}(\xi, \eta)+3 E_{13} \cos (\xi) \cos (\eta) \\
H_{x / y}(\xi, \eta) & =-\sqrt{3}\left(E_{15}-E_{16}\right) \sin (\xi) \sin (\eta) \\
H_{z^{2} / x^{2}}(\xi, \eta) & =2 E_{10} l_{2}(\xi, \eta) \\
H_{z^{2} / x y}(\xi, \eta) & =-2 \sqrt{3} E_{10} \sin (\xi) \sin (\eta) \\
H_{x^{2} / x y}(\xi, \eta) & =\sqrt{3}\left(E_{11}-E_{12}\right) \sin (\xi) \sin (\eta) \\
H_{x z / y z}(\xi, \eta) & =\sqrt{3}\left(E_{14}-E_{13}\right) \sin (\xi) \sin (\eta) \\
H_{z^{2} / x}(\xi, \eta) & =-2 \sqrt{3} E_{1} \sin (\xi) d_{1}(\xi, \eta) \\
H_{z^{2} / y}(\xi, \eta) & =2 E_{1} C_{2}(\xi, \eta) \\
H_{z^{2} / z}(\xi, \eta) & =E_{2} C_{1}(\xi, \eta) \\
H_{x^{2} / x}(\xi, \eta) & =-2 \sqrt{3}\left(\frac{1}{3} E_{5}-E_{3}\right) \sin (\xi) d_{1}(\xi, \eta) \\
H_{x^{2} / y}(\xi, \eta) & =-2 E_{3} C_{3}(\xi, \eta)-2 i E_{5} \cos (\xi) d_{1}(\xi, \eta) \\
H_{x^{2} / z}(\xi, \eta) & =-2 E_{4} C_{2}(\xi, \eta), \\
H_{x y / x}(\xi, \eta) & =-\frac{2}{3} E_{5} C_{3}(\xi, \eta)-6 i E_{3} \cos (\xi) d_{1}(\xi, \eta) \\
&
\end{aligned}
$$

$$
\begin{aligned}
& H_{x y / y}(\xi, \eta)=H_{x^{2} / x}(\xi, \eta) \\
& H_{x y / z}(\xi, \eta)=2 \sqrt{3} E_{4} \sin (\xi) d_{1}(\xi, \eta) \\
& H_{x z / x}(\xi, \eta)=\frac{2}{3} E_{6} C_{3}(\xi, \eta)+6 i E_{7} \cos (\xi) d_{1}(\xi, \eta) \\
& H_{x z / y}(\xi, \eta)=2 \sqrt{3}\left(\frac{1}{3} E_{6}-E_{7}\right) \sin (\xi) d_{1}(\xi, \eta) \\
& H_{x z / z}(\xi, \eta)=-2 \sqrt{3} E_{8} \sin (\xi) d_{1}(\xi, \eta) \\
& H_{y z / x}(\xi, \eta)=H_{x z / y}(\xi, \eta) \\
& H_{y z / y}(\xi, \eta)=2 E_{7} C_{3}(\xi, \eta)+2 i E_{6} \cos (\xi) d_{1}(\xi, \eta) \\
& H_{y z / z}(\xi, \eta)=2 E_{8} C_{2}(\xi, \eta)
\end{aligned}
$$

where

$$
\begin{aligned}
E_{1}= & \frac{1}{2}\left[-V_{p d \sigma}\left(\sin ^{2} \phi-\frac{1}{2} \cos ^{2} \phi\right)+\sqrt{3} V_{p d \pi} \sin ^{2} \phi\right] \\
& \times \cos \phi
\end{aligned}
$$

$$
E_{2}=\left[-V_{p d \sigma}\left(\sin ^{2} \phi-\frac{1}{2} \cos ^{2} \phi\right)-\sqrt{3} V_{p d \pi} \cos ^{2} \phi\right]
$$$$
\times \sin \phi \text {, }
$$

$$
\begin{aligned}
& E_{3}=\frac{1}{4}\left[\frac{\sqrt{3}}{2} V_{p d \sigma} \cos ^{3} \phi+V_{p d \pi} \cos \phi \sin ^{2} \phi\right], \\
& E_{4}=\frac{1}{2}\left[\frac{\sqrt{3}}{2} V_{p d \sigma} \sin \phi \cos ^{2} \phi-V_{p d \pi} \sin \phi \cos ^{2} \phi\right]
\end{aligned}
$$

$$
\begin{aligned}
& E_{5}=-\frac{3}{4} V_{p d \pi} \cos \phi \\
& E_{6}=-\frac{3}{4} V_{p d \pi} \sin \phi
\end{aligned}
$$

$E_{7}=\frac{1}{4}\left[-\sqrt{3} V_{p d \sigma} \cos ^{2} \phi-V_{p d \pi}\left(1-2 \cos ^{2} \phi\right)\right] \sin \phi$,

$$
E_{8}=\frac{1}{2}\left[-\sqrt{3} V_{p d \sigma} \sin ^{2} \phi-V_{p d \pi}\left(1-2 \sin ^{2} \phi\right)\right] \cos \phi,
$$

$$
\begin{gathered}
E_{9}=\frac{1}{4} V_{d d \sigma}+\frac{3}{4} V_{d d \delta} \\
E_{10}=-\frac{\sqrt{3}}{4}\left[V_{d d \sigma}-V_{d d \delta}\right] \\
E_{11}=\frac{3}{4} V_{d d \sigma}+\frac{1}{4} V_{d d \delta} \\
E_{12}=V_{d d \pi} \\
E_{13}=V_{d d \pi} \\
E_{14}=V_{d d \delta} \\
E_{15}=V_{p p \sigma} \\
E_{16}=V_{p p \pi} .
\end{gathered}
$$


Here the angle $\phi$ characterizes the structure of the unit cell of the compound and it is determined by purely geometric reasons (see Fig. 1). For the ideal trigonal prism structure, neglecting the marginal deviations from it in real systems, we have $\phi=\arccos [\sqrt{4 / 7}]$, so that $\cos \phi=\sqrt{4 / 7}$ and $\sin \phi=$ $\sqrt{3 / 7}$.

With these expressions, taking into account also the further changes of basis, the Hamiltonian at the $\Gamma$ point can be divided in sub-blocks as

$$
\begin{gathered}
\hat{H}_{\mathrm{E}}(\Gamma)=\left(\begin{array}{ccc}
\hat{H}_{z d_{0}}(\Gamma) & 0 & 0 \\
0 & \hat{H}_{p d_{2}}(\Gamma) & 0 \\
0 & 0 & \hat{H}_{p d_{2}}(\Gamma)
\end{array}\right), \\
\hat{H}_{\mathrm{O}}(\Gamma)=\left(\begin{array}{ccc}
\hat{H}_{p d_{1}}(\Gamma) & 0 & 0 \\
0 & \hat{H}_{p d_{1}}(\Gamma) & 0 \\
0 & 0 & \Gamma_{z}
\end{array}\right),
\end{gathered}
$$

where

$$
\begin{aligned}
& \hat{H}_{z d_{0}}(\Gamma)=\left(\begin{array}{cc}
\Gamma_{0} & \sqrt{2} \Gamma_{z d_{0}} \\
\sqrt{2} \Gamma_{z d_{0}} & \Gamma_{z}^{\mathrm{E}}
\end{array}\right), \\
& \hat{H}_{p d_{2}}(\Gamma)=\left(\begin{array}{cc}
\Gamma_{2} & \sqrt{2} \Gamma_{p d_{2}} \\
\sqrt{2} \Gamma_{p d_{2}} & \Gamma_{p}^{\mathrm{E}}
\end{array}\right), \\
& \hat{H}_{p d_{1}}(\Gamma)=\left(\begin{array}{cc}
\Gamma_{1} & \sqrt{2} \Gamma_{p d_{2}} \\
\sqrt{2} \Gamma_{p d_{2}} & \Gamma_{p}^{\mathrm{O}}
\end{array}\right) .
\end{aligned}
$$

The parameters $\Gamma_{\alpha}$ can be viewed as "molecular" energy levels, and the quantities $\Gamma_{\alpha, \beta}$ can be viewed as hybridization parameters. Their explicit expressions read

$$
\begin{gathered}
\Gamma_{0}=H_{z^{2} / z^{2}}(\Gamma)=\Delta_{0}+6 E_{9} \\
\Gamma_{1}=H_{x z / x z}(\Gamma)=H_{y z / y z}(\Gamma)=\Delta_{1}+3\left[E_{13}+E_{14}\right] \\
\Gamma_{2}=H_{x y / x y}(\Gamma)=H_{x^{2} / x^{2}}(\Gamma)=\Delta_{2}+3\left[E_{11}+E_{12}\right] \\
\Gamma_{p}^{\mathrm{E}}=\Gamma_{p}+V_{p p \pi} \\
\Gamma_{p}^{\mathrm{O}}=\Gamma_{p}-V_{p p \pi} \\
\Gamma_{z}^{\mathrm{E}}=\Gamma_{z}-V_{p p \sigma} \\
\Gamma_{z}^{\mathrm{O}}=\Gamma_{z}+V_{p p \sigma} \\
\Gamma_{p}=H_{x / x}(\Gamma)=H_{y / y}(\Gamma)=\Delta_{p}+3\left[E_{15}+E_{16}\right] \\
\Gamma_{z}=H_{z / z}(\Gamma)=\Delta_{z}+6 E_{16} \\
\Gamma_{z d_{0}}=H_{3 z^{2}-r^{2} / z}(\Gamma)=3 E_{2} \\
\Gamma_{p d_{2}}=H_{x^{2}-y^{2} / y}(\Gamma)=H_{x y / x}(\Gamma)=-2\left[3 E_{3}+E_{5}\right] \\
\Gamma_{p d_{1}}=H_{x z / x}(\Gamma)=H_{y z / y}(\Gamma)=2\left[3 E_{7}+E_{6}\right]
\end{gathered}
$$

At the $\mathrm{K}$ point, in the proper basis described in the main text, we can write the even and odd blocks of the
Hamiltonian as

$$
\begin{gathered}
\hat{H}_{\mathrm{E}}(\mathrm{K})=\left(\begin{array}{ccc}
\hat{H}_{p d_{0}}(\mathrm{~K}) & 0 & 0 \\
0 & \hat{H}_{z d_{2}}(\mathrm{~K}) & 0 \\
0 & 0 & \hat{H}_{p d_{2}}(\mathrm{~K})
\end{array}\right), \\
\hat{H}_{\mathrm{O}}=\left(\begin{array}{ccc}
\hat{H}_{p d_{1}}(\mathrm{~K}) & 0 & 0 \\
0 & \hat{H}_{z d_{1}}(\mathrm{~K}) & 0 \\
0 & 0 & K_{p}^{\mathrm{O}}
\end{array}\right),
\end{gathered}
$$

where

$$
\begin{gathered}
\hat{H}_{p d_{0}}(\mathrm{~K})=\left(\begin{array}{cc}
K_{0} & -2 i K_{p d_{0}} \\
2 i K_{p d_{0}} & K_{p}^{\mathrm{E}}
\end{array}\right), \\
\hat{H}_{z d_{2}}(\mathrm{~K})=\left(\begin{array}{cc}
K_{2} & 2 K_{z d_{2}} \\
2 K_{z d_{2}} & K_{z}^{\mathrm{E}}
\end{array}\right), \\
\hat{H}_{p d_{2}}(\mathrm{~K})=\left(\begin{array}{cc}
K_{2} & i \sqrt{8} K_{p d_{2}} \\
-i \sqrt{8} K_{p d_{2}} & K_{p}^{\mathrm{E}}
\end{array}\right), \\
\hat{H}_{p d_{1}}(\mathrm{~K})=\left(\begin{array}{cc}
K_{1} & \sqrt{8} K_{p d_{1}} \\
\sqrt{8} K_{p d_{1}} & K_{p}^{\mathrm{O}}
\end{array}\right), \\
\hat{H}_{z d_{1}}(\mathrm{~K})=\left(\begin{array}{cc}
K_{1} & -2 i K_{z d_{1}} \\
2 i K_{z d_{1}} & K_{z}^{\mathrm{O}}
\end{array}\right) .
\end{gathered}
$$

The parameters $K_{\alpha}, K_{\alpha, \beta}$ read here

$$
\begin{gathered}
K_{0}=H_{z^{2} / z^{2}}(\mathrm{~K})=\Delta_{0}-3 E_{9}, \\
K_{1}=H_{x z / x z}(\mathrm{~K})=H_{y z / y z}(\mathrm{~K})=\Delta_{1}-\frac{3}{2}\left[E_{13}+E_{14}\right],
\end{gathered}
$$

$$
K_{2}=H_{x y / x y}(\mathrm{~K})=H_{x^{2} / x^{2}}(\mathrm{~K})=\Delta_{2}-\frac{3}{2}\left[E_{11}+E_{12}\right],
$$

$$
\begin{aligned}
& K_{p}^{\mathrm{E}}=K_{p}+V_{p p \pi}, \\
& K_{p}^{\mathrm{O}}=K_{p}-V_{p p \pi}, \\
& K_{z}^{\mathrm{E}}=K_{z}-V_{p p \sigma}, \\
& K_{z}^{\mathrm{O}}=K_{z}+V_{p p \sigma},
\end{aligned}
$$$$
K_{p}=H_{x / x}(\mathrm{~K})=H_{y / y}(\mathrm{~K})=\Delta_{p}-\frac{3}{2}\left[E_{15}+E_{16}\right],
$$

$$
K_{z}=H_{z / z}(\mathrm{~K})=\Delta_{z}-3 E_{16},
$$

$$
K_{p d_{0}}=H_{3 z^{2}-r^{2} / y}(\mathrm{~K})=i H_{3 z^{2}-r^{2} / x}(\mathrm{~K})=-3 E_{1},
$$

$$
K_{z d_{2}}=H_{x^{2}-y^{2} / z}(\mathrm{~K})=i H_{x y / z}(\mathrm{~K})=3 E_{4},
$$

$$
K_{p d_{2}}=H_{x^{2}-y^{2} / y}(\mathrm{~K})=-H_{x y / x}(\mathrm{~K})=-i H_{x^{2}-y^{2} / x}(\mathrm{~K})
$$$$
=-i H_{x y / y}(\mathrm{~K})=\left[E_{5}-3 E_{3}\right] \text {, }
$$

$$
\begin{aligned}
K_{p d_{1}} & =H_{x z / x}(\mathrm{~K})=-H_{y z / y}(\mathrm{~K})=i H_{x z / y}(\mathrm{~K}) \\
& =i H_{y z / x}(\mathrm{~K})=\left[E_{6}-3 E_{7}\right], \\
K_{z d_{1}} & =H_{y z / z}(\mathrm{~K})=i H_{x z / z}(\mathrm{~K})=-3 E_{8} .
\end{aligned}
$$




\section{Interlayer hopping terms}

Interlayer hopping is ruled by the Slater-Koster parameters $U_{p p \sigma}, U_{p p \pi}$ describing hopping between S-3p orbitals belonging to different layers.

In terms of the reduced momentum variables $\xi=k_{x} a / 2$, $\eta=\sqrt{3} k_{y} a / 2$, we have thus

$$
\begin{aligned}
& I_{x / x}(\xi, \eta)=\frac{1}{2}\left[E_{19} C_{3}(\xi,-\eta)+i 3 E_{17} \cos \xi d_{1}(\xi,-\eta)\right], \\
& I_{y / y}(\xi, \eta)=\frac{1}{2}\left[E_{17} C_{3}(\xi,-\eta)+i 3 E_{19} \cos \xi d_{1}(\xi,-\eta)\right],
\end{aligned}
$$

$$
\begin{gathered}
I_{z / z}(\xi, \eta)=E_{18} C_{1}(\xi,-\eta), \\
I_{x / y}(\xi, \eta)=\frac{\sqrt{3}}{2}\left[E_{17}-E_{19}\right] \sin \xi d_{1}(\xi,-\eta), \\
I_{x / z}(\xi, \eta)=-\sqrt{3} E_{20} \sin \xi d_{1}(\xi, \eta) \\
I_{y / z}(\xi, \eta)=-E_{20} C_{2}(\xi,-\eta) \\
I_{z / z}(\xi, \eta)=E_{18} C_{1}(\xi,-\eta)
\end{gathered}
$$

where

$$
\begin{gathered}
E_{17}=U_{p p \sigma} \cos ^{2} \beta+U_{p p \pi} \sin ^{2} \beta, \\
E_{18}=U_{p p \sigma} \sin ^{2} \beta+U_{p p \pi} \cos ^{2} \beta, \\
E_{19}=U_{p p \pi}, \\
E_{20}=\left[U_{p p \sigma}-U_{p p \pi}\right] \cos \beta \sin \beta .
\end{gathered}
$$

Here $\beta$ is the angle between the line connecting the two $\mathrm{S}$ atoms with respect to the $\mathrm{S}$ planes (see Fig. 1). Denoting $w$ the distance between the two $\mathrm{S}$ planes, we have

$$
\begin{aligned}
& \cos \beta=\frac{a}{\sqrt{a^{2}+3 w^{2}}}, \\
& \sin \beta=\frac{\sqrt{3} w}{\sqrt{a^{2}+3 w^{2}}} .
\end{aligned}
$$

Using typical values for bulk $\mathrm{MoS}_{2}, a=3.16 \AA$, and $w=$ $2.975 \AA$, we get $\cos \beta=0.523$ and $\sin \beta=0.852$. At the highsymmetry points $\Gamma, \mathrm{K}$, we have thus

$$
\begin{aligned}
& \Gamma_{p p}=I_{x / x}(\Gamma)=I_{y / y}(\Gamma)=\frac{3}{2}\left[E_{19}+E_{17}\right], \\
\Gamma_{z z}=I_{z / z}(\Gamma)=3 E_{18} & \\
K_{p p}= & I_{x / x}(\mathrm{~K})=-I_{y / y}(\mathrm{~K})=-i I_{x / y}(\mathrm{~K})=-i I_{y / x}(\mathrm{~K}) \\
= & \frac{3}{4}\left[E_{19}-E_{17}\right] \\
K_{p z}= & I_{y / z}(\mathrm{~K})=I_{z / y}(\mathrm{~K}) \\
= & -i I_{x / z}(\mathrm{~K})=-i I_{z / x}(\mathrm{~K})=\frac{3}{2} E_{20} .
\end{aligned}
$$

\section{APPENDIX B: DECOMPOSITION OF THE HAMILTONIAN IN SUB-BLOCKS AT HIGH-SYMMETRY POINTS}

In this appendix we summarize the different unitary transformations that make it possible to decompose at special high-symmetry points the higher rank Hamiltonian matrix into smaller sub-blocks. In all the cases we treat in a separate way the "even" and "odd" blocks, namely electronics states with even and odd symmetry with respect to the $z \rightarrow-z$ inversion.

\section{Single layer \\ a. $\Gamma$ point}

In the Hilbert space defined by the vector basis $\tilde{\phi}_{k}^{\dagger}$ in Eq. (9), the even and odd blocks of the Hamiltonian can be written, respectively, as

$$
\hat{H}_{\mathrm{E}}(\Gamma)=\left(\begin{array}{cccccc}
\Gamma_{0} & 0 & 0 & 0 & 0 & \sqrt{2} \Gamma_{z d_{0}} \\
0 & \Gamma_{2} & 0 & 0 & \sqrt{2} \Gamma_{p d_{2}} & 0 \\
0 & 0 & \Gamma_{2} & \sqrt{2} \Gamma_{p d_{2}} & 0 & 0 \\
0 & 0 & \sqrt{2} \Gamma_{p d_{2}} & \Gamma_{p}^{\mathrm{E}} & 0 & 0 \\
0 & \sqrt{2} \Gamma_{p d_{2}} & 0 & 0 & \Gamma_{p}^{\mathrm{E}} & 0 \\
\sqrt{2} \Gamma_{z d_{0}} & 0 & 0 & 0 & 0 & \Gamma_{z}^{\mathrm{E}}
\end{array}\right)
$$

and

$$
\hat{H}_{\mathrm{O}}(\Gamma)=\left(\begin{array}{ccccc}
\Gamma_{1} & 0 & \sqrt{2} \Gamma_{p d_{1}} & 0 & 0 \\
0 & \Gamma_{1} & 0 & \sqrt{2} \Gamma_{p d_{1}} & 0 \\
\sqrt{2} \Gamma_{p d_{1}} & 0 & \Gamma_{p}^{\mathrm{O}} & 0 & 0 \\
0 & \sqrt{2} \Gamma_{p d_{1}} & 0 & \Gamma_{p}^{\mathrm{O}} & 0 \\
0 & 0 & 0 & 0 & \Gamma_{z}^{\mathrm{O}}
\end{array}\right) .
$$


The division in sub-blocks is already evident in Eqs. (B1) and (B2). They can be further ordered using the basis

$$
\bar{\phi}_{k}^{\dagger}=\left(\bar{\phi}_{k, z d_{0}}^{\dagger}, \bar{\phi}_{k, p d_{2}, y}^{\dagger}, \bar{\phi}_{k, p d_{2}, x}^{\dagger}, \bar{\phi}_{k, p d_{1}, x}^{\dagger}, \bar{\phi}_{k, p d_{1}, y}^{\dagger}, \bar{\phi}_{k, z}^{\dagger}\right),
$$

where

$$
\begin{aligned}
\bar{\phi}_{k, z d_{0}}^{\dagger}= & \left(d_{k, 3 z^{2}-r^{2}}^{\dagger}, p_{k, z, A}^{\dagger}\right), \\
\bar{\phi}_{k, p d_{2}, y}^{\dagger} & =\left(d_{k, x^{2}-y^{2}}^{\dagger}, p_{k, y, S}^{\dagger}\right), \\
\bar{\phi}_{k, p d_{2}, x}^{\dagger} & =\left(d_{k, x y}^{\dagger}, p_{k, x, S}^{\dagger}\right), \\
\bar{\phi}_{k, p d_{1}, x}^{\dagger} & =\left(d_{k, x z}^{\dagger}, p_{k, x, A}^{\dagger}\right), \\
\bar{\phi}_{k, p d_{1}, y}^{\dagger} & =\left(d_{k, y z}^{\dagger}, p_{k, y, A}^{\dagger}\right), \\
\bar{\phi}_{k, z}^{\dagger} & =\left(p_{k, z, S}^{\dagger}\right) .
\end{aligned}
$$

In this basis we get Eqs. (11) and (12), where

$$
\begin{aligned}
& \hat{H}_{z d_{0}}(\Gamma)=\left(\begin{array}{cc}
\Gamma_{0} & \sqrt{2} \Gamma_{z d_{0}} \\
\sqrt{2} \Gamma_{z d_{0}} & \Gamma_{z}^{\mathrm{E}}
\end{array}\right), \\
& \hat{H}_{p d_{2}}(\Gamma)=\left(\begin{array}{cc}
\Gamma_{2} & \sqrt{2} \Gamma_{p d_{2}} \\
\sqrt{2} \Gamma_{p d_{2}} & \Gamma_{p}^{\mathrm{E}}
\end{array}\right), \\
& \hat{H}_{p d_{1}}(\Gamma)=\left(\begin{array}{cc}
\Gamma_{1} & \sqrt{2} \Gamma_{p d_{1}} \\
\sqrt{2} \Gamma_{p d_{1}} & \Gamma_{p}^{\mathrm{O}}
\end{array}\right) .
\end{aligned}
$$

\section{b. K point}

In the basis defined by the Hilbert vector $\tilde{\phi}_{k}^{\dagger}$, the Hamiltonian at the $\mathrm{K}$ point reads, for the even and odd blocks, respectively,

$$
\begin{gathered}
\hat{H}_{\mathrm{E}}(\mathrm{K})=\left(\begin{array}{cccccc}
K_{0} & 0 & 0 & -i \sqrt{2} K_{p d_{0}} & \sqrt{2} K_{p d_{0}} & 0 \\
0 & K_{2} & 0 & i \sqrt{2} K_{p d_{2}} & \sqrt{2} K_{p d_{2}} & \sqrt{2} K_{z d_{2}} \\
0 & 0 & K_{2} & -\sqrt{2} K_{p d_{2}} & i \sqrt{2} K_{p d_{2}} & -i \sqrt{2} K_{z d_{2}} \\
i \sqrt{2} K_{p d_{0}} & -i \sqrt{2} K_{p d_{2}} & -\sqrt{2} K_{p d_{2}} & K_{p}^{\mathrm{E}} & 0 & 0 \\
\sqrt{2} K_{p d_{0}} & \sqrt{2} K_{p d_{2}} & -i \sqrt{2} K_{p d_{2}} & 0 & K_{p}^{\mathrm{E}} & 0 \\
0 & \sqrt{2} K_{z d_{2}} & i \sqrt{2} K_{z d_{2}} & 0 & 0 & K_{z}^{\mathrm{E}}
\end{array}\right), \\
\hat{H}_{\mathrm{O}}(\mathrm{K})=\left(\begin{array}{ccccc}
K_{1} & 0 & \sqrt{2} K_{p d_{1}} & -i \sqrt{2} K_{p d_{1}} & -i \sqrt{2} K_{z d_{1}} \\
0 & K_{1} & -i \sqrt{2} K_{p d_{1}} & -\sqrt{2} K_{p d_{1}} & \sqrt{2} K_{z d_{1}} \\
\sqrt{2} K_{p d_{1}} & i \sqrt{2} K_{p d_{1}} & K_{p}^{\mathrm{O}} & 0 & 0 \\
i \sqrt{2} K_{p d_{1}} & -\sqrt{2} K_{p d_{1}} & 0 & K_{p}^{\mathrm{O}} & 0 \\
i \sqrt{2} K_{z d_{1}} & \sqrt{2} K_{z d_{1}} & 0 & 0 & K_{z}^{\mathrm{O}}
\end{array}\right)
\end{gathered}
$$

In order to decouple the Hamiltonian, it is convenient to introduce the chiral basis defined by the vector $\bar{\psi}_{k}^{\dagger}$ in (17). In this Hilbert space we have thus

$$
\begin{aligned}
\hat{H}_{\mathrm{E}}(\mathrm{K}) & =\left(\begin{array}{ccc}
\hat{H}_{p d_{0}}(\mathrm{~K}) & 0 & 0 \\
0 & \hat{H}_{z d_{2}}(\mathrm{~K}) & 0 \\
0 & 0 & \hat{H}_{p d_{2}}(\mathrm{~K})
\end{array}\right), \\
\hat{H}_{\mathrm{O}} & =\left(\begin{array}{ccc}
\hat{H}_{p d_{1}}(\mathrm{~K}) & 0 & 0 \\
0 & \hat{H}_{z d_{1}}(\mathrm{~K}) & 0 \\
0 & 0 & K_{p}^{\mathrm{O}}
\end{array}\right)
\end{aligned}
$$

where

$$
\begin{gathered}
\hat{H}_{p d_{0}}(\mathrm{~K})=\left(\begin{array}{cc}
K_{0} & -i 2 K_{p d_{0}} \\
i 2 K_{p d_{0}} & K_{p}^{\mathrm{E}}
\end{array}\right), \\
\hat{H}_{z d_{2}}(\mathrm{~K})=\left(\begin{array}{cc}
K_{2} & 2 K_{p d_{2}} \\
2 K_{p d_{2}} & K_{z}^{\mathrm{E}}
\end{array}\right), \\
\hat{H}_{p d_{2}}(\mathrm{~K})=\left(\begin{array}{cc}
K_{2} & i \sqrt{8} K_{p d_{2}} \\
-i \sqrt{8} K_{p d_{2}} & K_{p}^{\mathrm{E}}
\end{array}\right),
\end{gathered}
$$

$$
\begin{aligned}
& \hat{H}_{p d_{1}}(\mathrm{~K})=\left(\begin{array}{cc}
K_{1} & \sqrt{8} K_{p d_{1}} \\
\sqrt{8} K_{p d_{1}} & K_{p}^{\mathrm{O}}
\end{array}\right), \\
& \hat{H}_{z d_{1}}(\mathrm{~K})=\left(\begin{array}{cc}
K_{1} & -i 2 K_{p d_{1}} \\
i 2 K_{p d_{1}} & K_{z}^{\mathrm{O}}
\end{array}\right) .
\end{aligned}
$$

\section{Bulk system}

The general structure of the TB Hamiltonian $H_{\text {bulk }}$ for the bulk system, using the basis defined in (23), is provided in Eqs. (26)-(31). We also remind here the symmetry property (25) that relates the matrix elements of $H_{2}$ to $H_{1}$.

As mentioned in the main text, for $k_{z}=0$ the band structure can still be divided in two independent blocks with even and odd symmetry with respect to the transformation $z \rightarrow-z$. $^{104}$ Further simplifications are encountered at the high-symmetry points $\Gamma$ and $\mathrm{K}$.

\section{a. $\Gamma$ point}

We first notice that at the $\Gamma$ point the relation (25) does not play any role, i.e., $\hat{H}_{2}(\Gamma)=\hat{H}_{1}(\Gamma)$, where $\hat{H}_{1}(\Gamma)$ is defined by Eqs. (10)-(12) in the main text. 
The Hamiltonian is thus completely determined by the interlayer hopping matrix $\hat{I}$ that at the $\Gamma$ point reads

$$
\hat{I}(\Gamma)=\left(\begin{array}{ccc}
\Gamma_{p p} & 0 & 0 \\
0 & \Gamma_{p p} & 0 \\
0 & 0 & \Gamma_{z z}
\end{array}\right) .
$$

A convenient basis to decouple the Hamiltonian into smaller sub-blocks is thus

$$
\bar{\Phi}_{k}^{\dagger}=\left(\bar{\Phi}_{k, z d_{0}}^{\dagger}, \bar{\Phi}_{k, p d_{2}, y}^{\dagger}, \bar{\Phi}_{k, p d_{2}, x}^{\dagger}, \bar{\Phi}_{k, p d_{1}, x}^{\dagger}, \bar{\Phi}_{k, p d_{1}, y}^{\dagger}, \bar{\Phi}_{k, z}^{\dagger}\right),
$$

where

$$
\begin{gathered}
\bar{\Phi}_{k, z d_{0}}^{\dagger}=\left(d_{k, 3 z^{2}-r^{2}, 1}^{\dagger}, p_{k, z, A, 1}^{\dagger}, d_{k, 3 z^{2}-r^{2}, 2}^{\dagger}, p_{k, z, A, 2}^{\dagger}\right), \\
\bar{\Phi}_{k, p d_{2}, y}^{\dagger}=\left(d_{k, x^{2}-y^{2}, 1}^{\dagger}, p_{k, y, S, 1}^{\dagger}, d_{k, x^{2}-y^{2}, 2}^{\dagger}, p_{k, y, S, 2}^{\dagger}\right), \\
\bar{\Phi}_{k, p d_{2}, x}^{\dagger}=\left(d_{k, x y, 1}^{\dagger}, p_{k, x, S, 1}^{\dagger}, d_{k, x y, 2}^{\dagger}, p_{k, x, S, 2}^{\dagger}\right), \\
\bar{\Phi}_{k, p d_{1}, x}^{\dagger}=\left(d_{k, x z, 1}^{\dagger}, p_{k, x, A, 1}^{\dagger}, d_{k, x z, 2}^{\dagger}, p_{k, x, A, 2}^{\dagger}\right), \\
\bar{\Phi}_{k, p d_{1}, y}^{\dagger}=\left(d_{k, y z, 1}^{\dagger}, p_{k, y, A, 1}^{\dagger}, d_{k, y z, 2}^{\dagger}, p_{k, y, A, 2}^{\dagger}\right), \\
\bar{\Phi}_{k, z}^{\dagger}=\left(p_{k, z, S, 1}^{\dagger}, p_{k, z, S, 1}^{\dagger}\right) .
\end{gathered}
$$

The resulting total Hamiltonian can be written as

$$
\hat{H}_{\text {bulk }}(\Gamma)=\left(\begin{array}{cc}
\hat{H}_{\mathrm{E}, \text { bulk }}(\Gamma) & 0 \\
0 & \hat{H}_{\mathrm{O}, \text { bulk }}(\Gamma)
\end{array}\right),
$$

where

$$
\begin{aligned}
\hat{H}_{\mathrm{E}, \text { bulk }}(\Gamma) & =\left(\begin{array}{ccc}
\hat{H}_{z d_{0}, \text { bulk }}(\Gamma) & 0 & 0 \\
0 & \hat{H}_{p d_{2}, \text { bulk }}(\Gamma) & 0 \\
0 & 0 & \hat{H}_{p d_{2}, \text { bulk }}(\Gamma)
\end{array}\right), \\
\hat{H}_{\mathrm{O}, \text { bulk }}(\Gamma) & =\left(\begin{array}{ccc}
\hat{H}_{p d_{1}, \text { bulk }}(\Gamma) & 0 & 0 \\
0 & \hat{H}_{p d_{1}, \text { bulk }}(\Gamma) & 0 \\
0 & 0 & \hat{H}_{z, \text { bulk }}(\Gamma)
\end{array}\right),
\end{aligned}
$$

and where

$$
\begin{aligned}
\hat{H}_{z d_{0}, \text { bulk }} & =\left(\begin{array}{cccc}
\Gamma_{0} & \sqrt{2} \Gamma_{z d_{0}} & 0 & 0 \\
\sqrt{2} \Gamma_{z d_{0}} & \Gamma_{z}^{\mathrm{E}} & 0 & \Gamma_{z z} \\
0 & 0 & \Gamma_{0} & \sqrt{2} \Gamma_{z d_{0}} \\
0 & \Gamma_{z z} & \sqrt{2} \Gamma_{z d_{0}} & \Gamma_{z}^{\mathrm{E}}
\end{array}\right), \\
\hat{H}_{p d_{2}, \text { bulk }} & =\left(\begin{array}{cccc}
\Gamma_{2} & \sqrt{2} \Gamma_{p d_{2}} & 0 & 0 \\
\sqrt{2} \Gamma_{p d_{2}} & \Gamma_{p}^{\mathrm{E}} & 0 & \Gamma_{p p} \\
0 & 0 & \Gamma_{2} & \sqrt{2} \Gamma_{p d_{2}} \\
0 & \Gamma_{p p} & \sqrt{2} \Gamma_{p d_{2}} & \Gamma_{p}^{\mathrm{E}}
\end{array}\right),
\end{aligned}
$$

$$
\hat{H}_{p d_{1}, \text { bulk }}=\left(\begin{array}{cccc}
\Gamma_{1} & \sqrt{2} \Gamma_{p d_{1}} & 0 & 0 \\
\sqrt{2} \Gamma_{p d_{1}} & \Gamma_{p}^{\mathrm{O}} & 0 & \Gamma_{p p} \\
0 & 0 & \Gamma_{1} & \sqrt{2} \Gamma_{p d_{1}} \\
0 & \Gamma_{p p} & \sqrt{2} \Gamma_{p d_{1}} & \Gamma_{p}^{\mathrm{O}}
\end{array}\right)
$$

$$
\hat{H}_{z, \text { bulk }}=\left(\begin{array}{ll}
\Gamma_{z}^{\mathrm{O}} & \Gamma_{z z} \\
\Gamma_{z z} & \Gamma_{z}^{\mathrm{O}}
\end{array}\right)
$$

\section{b. K point}

The treatment of the bulk Hamiltonian at the $\mathrm{K}$ point, in order to get a matrix clearly divided into blocks, is a bit less straightforward than at the $\Gamma$ point.

We first notice that the interlayer matrix, in the basis $\tilde{\Phi}_{k}^{\dagger}$, reads

$$
\hat{I}(\mathrm{~K})=\left(\begin{array}{ccc}
K_{p p} & i K_{p p} & i K_{p z} \\
i K_{p p} & -K_{p p} & K_{p z} \\
i K_{p z} & K_{p z} & 0
\end{array}\right)
$$

We then redefine the orbitals $d_{k, y z, 2}^{\dagger} \rightarrow \bar{d}_{k, y z, 2}^{\dagger}=-d_{k, y z, 2}^{\dagger}$, $p_{k, y, \alpha, 2}^{\dagger} \rightarrow \bar{p}_{k, y, \alpha, 2}^{\dagger}=-p_{k, y, \alpha, 2}^{\dagger}(\alpha=\mathrm{A}, \mathrm{S})$, in order to get, according with $(25), \hat{H}_{2}(\Gamma)=\hat{H}_{1}(\Gamma)$.

Following what was done for the single layer, we can also introduce here a chiral basis. After a further rearrangement of the vector elements, we define thus the convenient Hilbert space as

$$
\begin{aligned}
\bar{\Psi}_{k}^{\dagger}= & \left(\bar{\Psi}_{k, p z d_{0} 2, L}^{\dagger}, \bar{\Psi}_{k, p z d_{0} 2, R}^{\dagger}, \bar{\Psi}_{k, p d_{2}, \mathrm{E}}^{\dagger},\right. \\
& \left.\bar{\Psi}_{k, p z d_{1}, R}^{\dagger}, \bar{\Psi}_{k, p z d_{1}, L}^{\dagger}, \bar{\Psi}_{k, p d_{1}, \mathrm{O}}^{\dagger}\right),
\end{aligned}
$$

where

$$
\begin{gathered}
\bar{\Psi}_{k, p z d_{0} 2, L}^{\dagger}=\left(d_{k, 3 z^{2}-r^{2}, 1}^{\dagger}, p_{k, L, S, 1}^{\dagger}, d_{k, R, 2}^{\dagger}, p_{k, z, A, 2}^{\dagger}\right), \\
\bar{\Psi}_{k, p z d_{0} 2, R}^{\dagger}=\left(d_{k, 3 z^{2}-r^{2}, 2}^{\dagger}, p_{k, R, S, 2}^{\dagger}, d_{k, L, 1}^{\dagger}, p_{k, z, A, 1}^{\dagger}\right), \\
\bar{\Psi}_{k, p d_{2}, \mathrm{E}}^{\dagger}=\left(d_{k, R, 1}^{\dagger}, p_{k, R, S, 1}^{\dagger}, d_{k, L, 1}^{\dagger}, p_{k, L, S, 1}^{\dagger}\right), \\
\bar{\Psi}_{k, p z d_{1}, R}^{\dagger}=\left(d_{k, R, 1}^{\dagger}, p_{k, z, S, 1}^{\dagger}, p_{k, R, A, 2}^{\dagger}\right), \\
\bar{\Psi}_{k, p z d_{1}, R}^{\dagger}=\left(d_{k, L, 2}^{\dagger}, p_{k, z, S, 2}^{\dagger}, p_{k, L, A, 1}^{\dagger}\right), \\
\bar{\Psi}_{k, p d_{1}, \mathrm{O}}^{\dagger}=\left(d_{k, L, 1}^{\dagger}, p_{k, R, A, 1}^{\dagger}, d_{k, R, 2}^{\dagger}, p_{k, L, A, 2}^{\dagger}\right) .
\end{gathered}
$$

On this basis, the Hamiltonian can be once more written as

$$
\hat{H}_{\text {bulk }}(\mathrm{K})=\left(\begin{array}{cc}
\hat{H}_{\mathrm{E}}(\mathrm{K}) & 0 \\
0 & \hat{H}_{\mathrm{O}}(\mathrm{K})
\end{array}\right),
$$

where $\hat{H}_{\mathrm{E}}(\mathrm{K}), \hat{H}_{\mathrm{O}}(\mathrm{K})$ are defined in Eqs. (35)-(40) of the main text. 
${ }^{1}$ K. S. Novoselov, A. K. Geim, S. V. Morozov, D. Jiang, Y. Zhang, S. V. Dubonos, I. V. Gregorieva, and A. A. Firsov, Science 306, 666 (2004).

${ }^{2}$ K. S. Novoselov, D. Jiang, F. Schedin, T. J. Booth, V. V. Khotkivich, S. V. Morozov, and A. K. Geim, Proc. Natl. Acad. Sci. USA 102, 10451 (2005).

${ }^{3}$ Y. Zhang, Y.-W. Tan, H. L. Stormer, and P. Kim, Nature (London) 438, 201 (2005).

${ }^{4}$ L. Britnell, R. V. Gorbachev, R. Jalil, B. D. Belle, F. Schedin, A. Mishchenko, T. Georgiou, M. I. Katsnelson, L. Eaves, S. V. Morozov, N. M. R. Peres, J. Leist, A. K. Geim, K. S. Novoselov, and L. A. Ponomarenko, Science 335, 947 (2012).

${ }^{5}$ T. Li and G. Galli, J. Phys. Chem. C 111, 16192 (2007).

${ }^{6}$ S. Lebègue and O. Eriksson, Phys. Rev. B 79, 115409 (2009).

${ }^{7}$ K. F. Mak, C. Lee, J. Hone, J. Shan, and T. F. Heinz, Phys. Rev. Lett. 105, 136805 (2010).

${ }^{8}$ A. Splendiani, L. Sun, Y. Zhang, T. Li, J. Kim, C.-Y. Chim, G. Galli, and F. Wang, Nano Lett. 10, 1271 (2010).

${ }^{9}$ J. Feng, X. Qian, C.-W. Huang, and J. Li, Nat. Photon. 6, 866 (2012).

${ }^{10}$ P. Lu, X. Wu, W. Guo, and X. C. Zeng, Phys. Chem. Chem. Phys. 14, 13035 (2012).

${ }^{11}$ H. Pan and Y.-W. Zhang, J. Phys. Chem. C 116, 11752 (2012).

${ }^{12}$ H. Peelaers and C. G. Vande Walle, Phys. Rev. B 86, 241401 (2012).

${ }^{13}$ W. S. Yun, S. W. Han, S. C. Hong, I. G. Kim, and J. D. Lee, Phys. Rev. B 85, 033305 (2012).

${ }^{14}$ E. Scalise, M. Houssa, G. Pourtois, V. Afanas'ev, and A. Stesmans, Nano Res. 5, 43 (2012).

${ }^{15}$ E. Scalise, M. Houssa, G. Pourtois, V. Afanas'ev, and A. Stesmans, Physica E (2013), doi: 10.1016/j.physe.2012.07.029.

${ }^{16}$ Y. Li, Y.-1. Li, C. M. Araujo, W. Luo, and R. Ahuja, Catal. Sci. Technol. (2013), doi: 10.1039/C3CY00207A.

${ }^{17}$ M. Ghorbani-Asl, S. Borini, A. Kuc, and T. Heine, Phys. Rev. B 87, 235434 (2013).

${ }^{18}$ H. Shi, H. Pan, Y.-W. Zhang, and B. I. Yakobson, Phys. Rev. B 87, 155304 (2013).

${ }^{19}$ L. Hromodová, R. Martoňák, and E. Tosatti, Phys. Rev. B 87, 144105 (2013).

${ }^{20}$ S. Horzum, H. Sahin, S. Cahangirov, P. Cudazzo, A. Rubio, T. Serin, and F. M. Peeters, Phys. Rev. B 87, 125415 (2013).

${ }^{21}$ K. F. Mak, K. He, J. Shan, and T. F. Heinz, Nat. Nanotech. 7, 494 (2012).

${ }^{22}$ K. F. Mak, K. He, C. Lee, G. H. Lee, J. Hone, T. F. Heinz, and J. Shan, Nat. Mater. 12, 207 (2013).

${ }^{23}$ T. Cao, J. Feng, J. Shi, Q. Niu, and E. Wang, Nat. Commun. 3, 887 (2012).

${ }^{24}$ G. Sallen, L. Bouet, X. Marie, G. Wang, C. R. Zhu, W. P. Han, Y. Lu, P. H. Tan, T. Amand, B. L. Liu, and B. Urbaszek, Phys. Rev. B 86, 081301 (2012).

${ }^{25}$ D. Xiao, G.-B. Liu, W. Feng, X. Xu, and W. Yao, Phys. Rev. Lett. 108, 196802 (2012).

${ }^{26}$ S. Wu, J. S. Ross, G.-B. Liu, G. Aivazian, A. Jones, Z. Fei, W. Zhu, D. Xiao, W. Yao, D. Cobden, and X. Xu, Nat. Phys. 9, 149 (2013).

${ }^{27}$ H. Zheng, J. Dai, W. Yao, D. Xiao, and X. Cui, Nat. Nanotechnol. 7, 490 (2012).

${ }^{28}$ H. Ochoa and R. Roldán, Phys. Rev. B 87, 245421 (2013).

${ }^{29}$ S. Tongay, J. Zhou, C. Ataca, K. Lo, T. S. Matthews, J. Li, J. C. Grossman, and J. Wu, Nano Lett. 12, 5576 (2012).
${ }^{30}$ P. R. Wallace, Phys. Rev. 71, 622 (1947).

${ }^{31}$ S. Reich, J. Maultzsch, C. Thomsen, and P. Ordejón, Phys. Rev. B 66, 035412 (2002).

${ }^{32}$ For a review, see, for instance, A. H. Castro Neto, F. Guinea, N. M. R. Peres, K. S. Novoselov, and A. K. Geim, Rev. Mod. Phys. 81, 109 (2009).

${ }^{33}$ E. McCann and M. Koshino, Rep. Prog. Phys. 76, 056503 (2013).

${ }^{34}$ J. Nilsson, A. H. Castro Neto, F. Guinea, and N. M. R. Peres, Phys. Rev. Lett. 97, 266801 (2006).

${ }^{35}$ B. Partoens and F. M. Peeters, Phys. Rev. B 74, 075404 (2006).

${ }^{36}$ B. Partoens and F. M. Peeters, Phys. Rev. B 75, 193402 (2007).

${ }^{37}$ A. A. Avetisyan, B. Partoens, and F. M. Peeters, Phys. Rev. B 79, 035421 (2009); 80, 195401 (2009); 81, 115432 (2010).

${ }^{38}$ M. Koshino and E. McCann, Phys. Rev. B 79, 125443 (2009).

${ }^{39}$ M. Koshino, Phys. Rev. B 81, 125304 (2010).

${ }^{40}$ M. Koshino and E. McCann, Phys. Rev. B 87, 045420 (2013).

${ }^{41}$ F. Zhang, B. Sahu, H. Min, and A. H. MacDonald, Phys. Rev. B 82, 035409 (2010).

${ }^{42}$ S. Yuan, R. Roldán, and M. I. Katsnelson, Phys. Rev. B 84, 125455 (2011).

${ }^{43}$ J.-A. Yan, W. Y. Ruan, and M. Y. Chou, Phys. Rev. B 83, 245418 (2011).

${ }^{44}$ R. Olsen, R. van Gelderen, and C. M. Smith, Phys. Rev. B 87, 115414 (2013).

${ }^{45}$ K. F. Mak, M. Y. Sfeir, J. A. Misewich, and T. F. Heinz, Proc. Natl. Acad. Sci. USA 107, 14999 (2010).

${ }^{46}$ C. H. Lui, Z. Q. Li, K. F. Mak, E. Cappelluti, and T. F. Heinz, Nat. Phys. 7, 944 (2011).

${ }^{47}$ W. Bao, L. Jing, J. Velasco, Jr., Y. Lee, G. Liu, D. Tran, B. Standley, M. Aykol, S. B. Cronin, D. Smirnov, M. Koshino, E. McCann, M. Bockrath, and C. N. Lau, Nat. Phys. 7, 948 (2011).

${ }^{48}$ N. Ubrig, P. Blake, D. van der Marel, and A. B. Kuzmenko, Europhys. Lett. 100, 58003 (2012).

${ }^{49}$ J. M. Soler, E. Artacho, J. Gale, A. García, J. Junquera, P. Ordejón, and D. Sánchez-Portal, J. Phys.: Condens. Matter 14, 2745 (2002).

${ }^{50}$ E. Artacho, E. Anglada, O. Dieguez, J. D. Gale, A. García, J. Junquera, R. M. Martin, P. Ordejón, J. M. Pruneda, D. SánchezPortal, and J. M. Soler, J. Phys.: Condens. Matter 20, 064208 (2008)

${ }^{51}$ M. S. Fuhrer, J. Nygård, L. Shih, M. Forero, Y.-G. Yoon, M. S. C. Mazzoni, H. J. Choi, J. Ihm, S. G. Louie, A. Zettl, and P. L. McEuen, Science 288, 494 (2000).

${ }^{52}$ B. Wang, M.-L. Bocquet, S. Marchini, S. Gunther, and J. Wintterlin, Phys. Chem. Chem. Phys. 10, 3530 (2008).

${ }^{53}$ P. O. Lehtinen, A. S. Foster, Y. C. Ma, A. V. Krasheninnikov, and R. M. Nieminen, Phys. Rev. Lett. 93, 187202 (2004).

${ }^{54}$ F. D. Novaes, R. Rurali, and P. Ordejón, ACS Nano 4, 7596 (2010)

${ }^{55}$ J. H. Ho, Y. H. Lai, Y. H. Chiu, and M. F. Lin, Nanotech. 19, 035712 (2008).

${ }^{56}$ J. J. Palacios, J. Fernandez-Rossier, and L. Brey, Phys. Rev. B 77, 195428 (2008).

${ }^{57}$ A. L. C. Pereira and P. A. Schulz, Phys. Rev. B 78, 125402 (2008).

${ }^{58}$ A. Carpio, L. L. Bonilla, F. de Juan, and M. A. H. Vozmediano, New J. Phys. 10, 053021 (2008).

${ }^{59}$ M. P. López-Sancho, F. de Juan, and M. A. H. Vozmediano, Phys. Rev. B 79, 075413 (2009).

${ }^{60}$ R. M. Ribeiro, V. M. Pereira, N. M. R. Peres, P. R. Briddon, and A. H. Castro Neto, New J. Phys. 11, 115002 (2009). 
${ }^{61}$ S. Yuan, R. Roldán, and M. I. Katsnelson, Phys. Rev. B 84, 035439 (2011).

${ }^{62}$ M. Neek-Amal, L. Covaci, and F. M. Peeters, Phys. Rev. B 86, 041405 (2012).

${ }^{63}$ S. Yuan, R. Roldán, A.-P. Jauho, and M. I. Katsnelson, Phys. Rev. B 87, 085430 (2013).

${ }^{64}$ N. Leconte, J. Moser, P. Ordejón, H. Tao, A. Lherbier, A. Bachtold, F. Alsina, C. M. Sotomayor-Torres, J.-C. Charlier, and S. Roche, ACS Nano 4, 4033 (2010).

${ }^{65}$ D. Soriano, N. Leconte, P. Ordejón, J.-C. Charlier, J. J. Palacios, and S. Roche, Phys. Rev. Lett. 107, 016602 (2011).

${ }^{66}$ N. Leconte, D. Soriano, S. Roche, P. Ordejón, J.-C. Charlier, and J. J. Palacios, ACS Nano 5, 3987 (2011).

${ }^{67}$ D. Van Tuan, A. Kumar, S. Roche, F. Ortmann, M. F. Thorpe, and P. Ordejón, Phys. Rev. B 86, 121408(R) (2012).

${ }^{68}$ H. Zheng, Z. F. Wang, T. Luo, Q. W. Shi, and J. Chen, Phys. Rev. B 75, 165414 (2007).

${ }^{69}$ F. Guinea, M. I. Katsnelson, and M. A. H. Vozmediano, Phys. Rev. B 77, 075422 (2008).

${ }^{70}$ E. V. Castro, N. M. R. Peres, and J. M. B. Lopes dos Santos, J. Optoelectron. Adv. Mater. 10, 1716 (2008).

${ }^{71}$ E. V. Castro, M. P. López-Sancho, and M. A. H. Vozmediano, New J. Phys. 11, 095017 (2009).

${ }^{72}$ E. V. Castro, M. P. López-Sancho, and M. A. H. Vozmediano, Phys. Rev. Lett. 104, 036802 (2010).

${ }^{73}$ E. V. Castro, M. P. López-Sancho, and M. A. H. Vozmediano, Phys. Rev. B 84, 075432 (2011).

${ }^{74}$ A. Cresti, N. Nemec, B. Biel, G. Niebler, F. Triozon, G. Cuniberti, and S. Roche, Nano Res. 1, 361 (2008).

${ }^{75}$ Y. C. Huang, C. P. Chang, W. S. Su, and M. F. Lin, J. Appl. Phys. 106, 013711 (2009).

${ }^{76}$ Y. Klymenko and O. Shevtsov, Eur. Phys. J. B 69, 383 (2009).

${ }^{77}$ J. M. B. Lopes dos Santos, N. M. R. Peres, and A. H. Castro Neto, Phys. Rev. Lett. 99, 256802 (2007).

${ }^{78}$ E. J. Mele, Phys. Rev. B 81, 161405 (2010).

${ }^{79}$ E. J. Mele, J. Phys. D: Appl. Phys. 45, 154004 (2012).

${ }^{80}$ S. Shallcross, S. Sharma, E. Kandelaki, and O. A. Pankratov, Phys. Rev. B 81, 165105 (2010).

${ }^{81}$ E. Suárez Morell, J. D. Correa, P. Vargas, M. Pacheco, and Z. Barticevic, Phys. Rev. B 82, 121407 (2010).

${ }^{82}$ R. de Gail, M. O. Goerbig, F. Guinea, G. Montambaux, and A. H. Castro Neto, Phys. Rev. B 84, 045436 (2011).

${ }^{83}$ R. Bistritzer and A. H. MacDonald, Proc. Natl. Acad. Sci. USA 108, 12233 (2011).

${ }^{84}$ E. Suárez Morell, P. Vargas, L. Chico, and L. Brey, Phys. Rev. B 84, 195421 (2011).

${ }^{85}$ P. Moon and M. Koshino, Phys. Rev. B 85, 195458 (2012).

${ }^{86}$ P. Moon and M. Koshino, Phys. Rev. B 87, 205404 (2013).
${ }^{87}$ P. San-José, J. González, and F. Guinea, Phys. Rev. Lett. 108, 216802 (2012).

${ }^{88}$ C. J. Tabert and E. J. Nicol, Phys. Rev. B 87, 121402 (2013).

${ }^{89}$ K. Kaasbjerg, K. S. Thygesen, and K. W. Jacobsen, Phys. Rev. B 85, 115317 (2012).

${ }^{90}$ K. Kaasbjerg, A.-P. Jauho, and K. S. Thygesen, Phys. Rev. B 87, 235312 (2013).

${ }^{91}$ W. Feng, Y. Yao, W. Zhu, J. Zhou, W. Yao, and D. Xiao, Phys. Rev. B 86, 165108 (2012).

${ }^{92}$ E. S. Kadantsev and P. Hawrylak, Solid State Commun. 152, 909 (2012).

${ }^{93}$ K. Kośmider and J. Fernández-Rossier, Phys. Rev. B 87, 075451 (2013).

${ }^{94}$ N. Zibouche, A. Kuc, and T. Heine, Eur. Phys. J. 85, 49 (2012).

${ }^{95}$ X. Li, J. T. Mullen, Z. Jin, K. M. Borysenko, M. Buongiorno Nardelli, and K. W. Kim, Phys. Rev. B 87, 115418 (2013).

${ }^{96}$ R. A. Bromley, R. B. Murray, and A. D. Yoffe, J. Phys. C: Solid State Phys. 5, 759 (1972).

${ }^{97}$ A. Kormanyos, V. Zolyomi, N. D. Drummond, P. Rakyta, G. Burkard, and V. I. Fal'ko, Phys. Rev. B 88, 045416 (2013).

${ }^{98}$ H. Rostami, A. G. Moghaddam, and R. Asgari, arXiv:1302.5901.

${ }^{99}$ F. Zahid, L. Liu, Y. Zhu, J. Wang, and H. Guo, AIP Adv. 3, 052111 (2013).

${ }^{100}$ J. C. Slater and G. F. Koster, Phys. Rev. 94, 1498 (1954).

${ }^{101}$ D. M. Ceperley and B. J. Alder, Phys. Rev. Lett. 45, 566 (1980).

${ }^{102}$ J. P. Perdew and A. Zunger, Phys. Rev. B 23, 5048 (1981).

${ }^{103}$ E. Artacho, D. Sánchez-Portal, P. Ordejón, A. García, and J. M. Soler, Phys. Status Solidi B 215, 809 (1999).

${ }^{104}$ N. J. Doran, B. Ricco, D. J. Titterington, and G. Wexler, J. Phys. C: Solid State Phys. 11, 685 (1978).

${ }^{105}$ W. H. Press, S. A. Teukolsky, W. T. Vetterling, and B. P. Flannery, Numerical Recipes (Cambridge University Press, Cambridge, 1992).

${ }^{106}$ A good fitting agreement with DFT data was shown in Ref. 99, but using a larger, nonorthogonal basis set and involving up to 96 fitting parameters.

${ }^{107}$ Note that, while the identification of the band splitting is relatively easy in the TB model due to the reduced number of bands, such a task is more delicate in the DFT band structure because of the high number of bands and of the sizable hybridization between different orbitals in not high-symmetry points of the Brillouin zone. In such context the concept of band splitting at the Q point in DFT is relatively ambiguous. As a qualitative estimate, we took thus as band splitting the energy split $1.36 \mathrm{eV}$ at the $\mathrm{Q}$ point between the lowest energy conduction band and the fifth band, which a careful analysis shows to have the closer orbital content than the lowest energy one. 